\title{
Multi-scalar-tensor equivalents for modified gravitational actions
}

\author{
Ahmet Baykal, * * and Özgür Delice ${ }^{2, \text { 甲 }}$ \\ ${ }^{1}$ Department of Physics, Faculty of Science and Letters, Niğde University, 51240 Niğde, Turkey \\ ${ }^{2}$ Department of Physics, Faculty of Science and Letters, Marmara University, 34722 Istanbul, Turkey
}

(Dated: June 29, 2021)

\begin{abstract}
A general scheme of constructing scalar-tensor equivalents to modified gravitational actions are studied using the algebra of exterior differential forms and the first order formalism that allows an independent connection and coframe. By introducing appropriate constraints on the connection, pseudo-Riemannian cases as well as non-Riemannian cases are discussed for various gravitational models. The issue of the dynamical degree of freedom for the resulting scalar fields is discussed at the level of the field equations. Explicit scalar-tensor equivalents for gravitational models based on $f(R)$ models, the quadratic curvature Lagrangians and the models involving the gradients of the scalar curvature are presented. In particular, explicit scalar-tensor equivalence for gravitational Lagrangians popular in some cosmological models are constructed.

PACS numbers: 04.20.Fy, 04.50.Kd
\end{abstract}

\section{INTRODUCTION}

Einstein's theory of general relativity is a well tested theory explaining the gravitational interaction ranging from weak to strong fields and from solar system scale to the whole Universe. However, its alternatives are taking a lot of interest recently. For an extensive survey of several motivations and various aspects of modified gravity theories, we refer to the recent reviews [1, 2] and the references therein. Actually, apart from mathematical curiosity to understand mathematical and physical properties of these theories by considering and comparing to the alternative theories, there are several physical motivations. For example, the quantum effects of gravity seem to require higher order curvature correction terms in the theory. In particular, the gravitational models based on scalars constructed from the terms that are quadratic in curvature components are motivated in different contexts ranging from low energy limit of string theories [3] , quantum theory of gravity [4, [5] , to viable cosmological models [6]. One of the popular alternative theories is based on $f(R)$ Lagrangian which replaces the usual Ricci scalar term $R$ in the Einstein-Hilbert action with an arbitrary algebraic function of $R$. The theories with corresponding Lagrangian involving functions of other curvature scalars or collections of those scalars, in Riemannian or more general contexts are also studied extensively.

An important feature of modified gravitational Lagrangians is the concept of scalar-tensor (ST) equivalence for these models. For instance, $f(R)$ theory is known to have equivalent Brans-Dicke-type scalar-tensor theory with an extra potential term for the scalar field. Actually, scalar-tensor theories that are equivalent to particular $f(R)$ models were first introduced long ago [7, 8]

\footnotetext{
* abaykal@nigde.edu.tr

† ozgur.delice@marmara.edu.tr
}

and since then it is reintroduced, studied and has been made use of intermittently to this day. ST equivalent of $f(R)$ models in different approaches has been introduced both in metric and in Palatini approaches. In particular, in a first order theory where connection and metric are treated as independent, in the Brans-Dicke-type theories [9], the scalar field is known to generate an algebraic torsion [10] and thus it can be cast into pure metric theory by eliminating torsion [11]. By means of the ST equivalence, these considerations also apply to generic $f(R)$ models as well. The ST equivalence of $f(R)$ models has also helped to investigate various aspects of $f(R)$ theories, such as the chameleon mechanism in $f(R)$ theories [12], the study of a Birkoff-Jebsen like theorem in generic $f(R)$ theories [13, 14], and the search for gravitationalwave solutions of modified gravity models [15].

In higher order metric theories of gravity, the Legendre transformation was previously introduced in [16, 17] for Lagrangian densities depending on scalar curvature and in particular Ricci tensor in a nonlinear way. Later, this work is extended to study the general quadratic curvature Lagrangian density of the form

$$
\mathcal{L}=\left(a R^{2}+b R^{\alpha \beta} R_{\alpha \beta}+c R^{\alpha \beta \mu \nu} R_{\alpha \beta \mu \nu}\right) * 1,
$$

where the Legendre transformation with respect to tensorial quantities was introduced in a more general mathematical setting [18]. Later, the ST equivalence is extended to Palatini-type modified gravity theories where metric and connection are treated as independent gravitational variables 19]. More recently, the Legendre transformation of modified gravity is revisited [20] in the context of ST equivalents of models based on $R+f(G)$ [21], to study Gibbons-Hawking surface terms of dynamically equivalent theories.

Meanwhile, the idea of multi-scalar-tensor equivalents were reintroduced in the study of particle content of particular modified gravitational models based on $f\left(R, R_{\mu \nu} R^{\mu \nu}, R_{\mu \nu \alpha \beta} R^{\mu \nu \alpha \beta}\right)$, to show that such modified theories share the common undesirable feature of spin2 ghosts with generic quadratic curvature models [22]. 
The present work can be considered as an extension of the multi-scalar-tensor equivalents, which are presented at the level of action, to the level of field equations for the physically relevant case studied in 22] as well as others. Thus, one of the motivations for the present work is to show that the mathematical formulation of the BransDicke type ST equivalence for generic $f(R)$ theories can naturally be extended to obtain the ST equivalents for a wider set of modified Lagrangians based on arbitrary curvature scalars in the general form indicated above. Such extensions are presented explicitly using, in particular, the curvature scalars built out of the quadratic curvature scalars for the sake of the simplicity of the presentation as well as regarding the application to general theory of relativity.

The multi-scalar-tensor equivalence for generic modified Lagrangians is studied extensively in [23] regarding the number of scalar fields involved where the multiscalar-tensor equivalents are constructed using the properties of the Legendre transform with constraints. As will explicitly be illustrated in the study of ST equivalents for various gravitational actions below, the dynamical degree of freedom in an ST equivalent for a gravitational model based on a function of a collection of several curvature scalars depends on the form of the function and the total number of independent scalar fields is related to the rank of the Hessian matrix of the Legendre transformation used in the construction of ST equivalents [23]. The present work, on the other hand, addresses the issue of the dynamical degree of freedom corresponding to the scalar fields in the resulting ST-equivalent model. For a given modified gravitational Lagrangian, the dynamical degree of freedom carried by the scalar fields can be studied at the level of the corresponding field equations and the dynamics of the scalar fields is not apparent at the level of action. For this reason, some explicit models involving modified quadratic curvature Lagrangians are presented since such gravitational models have important cosmological features. For example, they provide a framework for inflation [5], or else, in models with nonlinear curvature terms, it is possible to avoid cosmological singularity [24]. Later, versions of these models are also studied in order to account for the late-time acceleration of the Universe as a viable alternative to dark energy models [25].

The outline of the paper is as follows. In the next section, the first order constrained formalism is reviewed using the algebra of the exterior forms relative to an orthonormal coframe. The exterior differential form notation and the geometrical conventions used are adopted from 26 28]. In the third section, some important features of the scalar-tensor equivalence of $f(R)$ theory are briefly presented in both pseudo-Riemannian and non-Riemannian context with an independent connection having a nonvanishing torsion and a nonmetricity. The ST equivalence is then slightly generalized to a generic modified Lagrangian depending on an arbitrary contraction of Riemann tensor and the equivalence is presented at the level of the field equations and also a criterion for the resulting scalar field to be dynamical is given following the study of the Lagrange multiplier term of the generic modified Lagrangian. In the fourth section, the ST equivalence of gravitational Lagrangians of the form $f(R, Q, P, K, S)$ is presented in some generality with $Q, P, K, S$ being the quadratic curvature scalars defined below. Subsequently, ST equivalence of various modified gravitational Lagrangians, such as modified Ricci or Weyl square gravity, which could have important applications to cosmology and to the study of black holes are investigated in detail as applications of this equivalence scheme. Moreover, modified gravitational Lagrangians popular in cosmology involving inverse powers of quadratic curvature terms are shown to be dynamically equivalent to simpler quadratic curvature gravity models with nonminimal scalar couplings. As the last application, the ST equivalence for modified 6th order gravitational Lagrangians involving the derivatives of the scalar curvature is presented. The paper concludes with brief comments on the general features of the ST equivalence. A discussion of the relation between the equations we have discussed in the orthonormal coframe and their coordinate frame expressions is presented in the Appendix.

\section{FIELD EQUATIONS RELATIVE TO AN ORTHONORMAL COFRAME}

In this section the scheme of calculation of variational derivatives relative to an orthonormal coframe will briefly be presented [29, 31 33]. In the subsequent sections, the general formulas of this section will be applied to all the modified gravitational actions considered below. Using the language of differential forms defined on pseudoRiemannian manifolds, the basic independent gravitational variables that will be used below are defined in this section. In terms of the local basis, coframe 1-forms are denoted by $\left\{\theta^{\alpha}\right\}$ and the metric tensor takes the form $g=\eta_{\alpha \beta} \theta^{\alpha} \otimes \theta^{\beta}$ with $\eta_{\alpha \beta}=\operatorname{diag}(-+++)$. The exterior product of the basis 1-forms will be abbreviated as $\theta^{\alpha} \wedge \theta^{\beta} \wedge \cdots \equiv \theta^{\alpha \beta \cdots}$. The covariant exterior derivative $D$ acts on tensor-valued forms. The torsion 2-form $\Theta^{\alpha}=\frac{1}{2} T_{\beta \mu}^{\alpha} \theta^{\beta \mu}$ can be defined as

$$
\Theta^{\alpha}=D \theta^{\alpha}=d \theta^{\alpha}+\omega_{\beta}^{\alpha} \wedge \theta^{\beta},
$$

where $T_{\beta \mu}^{\alpha}$ are the components of the torsion tensor and $\omega^{\alpha}{ }_{\beta}$ are the connection 1-forms. Relative to an orthonormal coframe, metric compatibility for the connection 1forms reads $\omega_{\alpha \beta}+\omega_{\beta \alpha}=0$. In terms of the curvature 2 -forms $\Omega^{\alpha}{ }_{\beta}$, the Cartan's second structure equation has the form

$$
\Omega_{\beta}^{\alpha}=\frac{1}{2} R_{\beta \mu \nu}^{\alpha} \theta^{\mu \nu}=d \omega_{\beta}^{\alpha}+\omega_{\mu}^{\alpha} \wedge \omega_{\beta}^{\mu},
$$

where $R_{\beta \mu \nu}^{\alpha}$ are the components of the Riemann tensor in the orthonormal frame. The contractions of the 
forms and tensor-valued forms are defined with the help of the contraction operator $i_{e_{\alpha}} \equiv i_{\alpha}$ where $e_{\alpha}$ is the basis frame fields that are metric dual to the basis 1forms: $i_{\alpha} \theta^{\beta}=\delta_{\alpha}^{\beta}$. The symbol $*$ corresponds to the Hodge dual operator that defines an inner product for two $p$-forms in terms of the metric tensor. In terms of the Hodge dual, the oriented volume element reads $* 1=\sqrt{|g|} d x^{0} \wedge \cdots \wedge d x^{3}=\frac{1}{4 !} \epsilon_{\alpha \beta \mu \nu} \theta^{\alpha \beta \mu \nu}$ in four spacetime dimensions where $\epsilon_{\alpha \beta \mu \nu}$ is a completely antisymmetric permutation symbol. Ricci 1-forms can be defined as $R^{\alpha} \equiv i_{\beta} \Omega^{\beta \alpha}=R_{\beta \mu}^{\beta \alpha} \theta^{\mu}$, whereas the scalar curvature $R$ can be written as $R=i_{\alpha} R^{\alpha}$. All the other geometrical objects will be introduced as they are required in terms of the quantities defined here.

The gravitational Lagrangians will be assumed to depend on the set of basic gravitational variables $\left\{\theta^{\alpha}\right\}$ and $\left\{\omega^{\alpha}{ }_{\beta}\right\}$ as well as their exterior derivatives, namely $d \theta^{\alpha}$ and $d \omega^{\alpha}{ }_{\beta}$, through the scalars constructed from the contractions of the curvature tensor. In a more general framework, minimal coupling of matter fields requires that the matter Lagrangians involve $\theta^{\alpha}$ and $\omega^{\alpha}{ }_{\beta}$ but not $d \theta^{\alpha}$ and $d \omega^{\alpha}{ }_{\beta}$ [29]. Local Lorentz invariance of the gravitational Lagrangian forbids the explicit dependence of $\mathcal{L}$ on connection 1-forms and that the connection 1-forms enter into total Lagrangian via tensorial expressions and covariant exterior derivative. Moreover, in place of $d \theta^{\alpha}$ and $d \omega^{\alpha}{ }_{\beta}$ it is convenient to have the two forms $\Theta^{\alpha}$ and $\Omega^{\alpha}{ }_{\beta}$ since general gravitational Lagrangians studied here are mainly based on scalars built on the curvature 2forms. In the explicit examples below, the Lagrangians will turn out to depend on particular nonminimally coupled scalar fields as well. For the sake of simplicity, the dependence on matter fields will be omitted. In deriving the metric field equations for gravitational models, the first order formalism where coframe $\left\{\theta^{\alpha}\right\}$ and connection 1-forms $\left\{\omega^{\alpha}{ }_{\beta}\right\}$ are regarded as the gravitational variables, will be used [28, 29]. In this framework, the equations for the pseudo-Riemannian metric are derived from the coframe variation of the Lagrangian subject to the constraint that the torsion 2 -form $\Theta^{\alpha}=D \theta^{\alpha}$ vanishes. The metric compatibility of the connection 1-form $\omega_{\alpha \beta}+\omega_{\beta \alpha}=0$ is an algebraic constraint on the independent connection and it can simply be implemented into the total variational derivative by antisymmetrization of the coefficients of $\delta \omega_{\alpha \beta}$. However, the torsionfree constraint, namely $\Theta^{\alpha}=0$, is a dynamical constraint and can be imposed by extending the original Lagrangian density to include a Lagrange multiplier 2form term $\mathcal{L}_{L M}=\lambda_{\alpha} \wedge \Theta^{\alpha}$ as

$$
\mathcal{L}_{e}\left[\theta^{\alpha}, \Omega^{\alpha}{ }_{\beta}, \Theta^{\alpha}, \lambda_{\alpha}\right]=\mathcal{L}\left[\theta^{\alpha}, \Omega^{\alpha}{ }_{\beta}\right]+\lambda_{\alpha} \wedge \Theta^{\alpha} .
$$

In the study of modified gravitational field equations relative to an orthonormal coframe, the use of derivatives of Lagrangian 4-forms with respect to $p$-form fields are very convenient for the manipulation of the variational derivatives. Although it will not be made use of in the discussions below, it is possible to relate the derivatives of the volume form with respect to a $p$-form to partial derivatives of appropriate scalars with respect to components of $p$-forms, we refer the reader to [29]. By using the variational derivatives of the curvature and torsion 2 -forms

$$
\delta \Theta^{\alpha}=D \delta \theta^{\alpha}+\delta \omega_{\beta}^{\alpha} \wedge \theta^{\beta}, \quad \delta \Omega_{\beta}^{\alpha}=D \delta \omega_{\beta}^{\alpha},
$$

the total variational derivative of extended Lagrangian density $\mathcal{L}_{e}$ with respect to the independent variables can be found to have the form

$$
\begin{aligned}
\delta \mathcal{L}_{e} & =\delta \theta^{\alpha} \wedge\left(\frac{\partial \mathcal{L}_{e}}{\partial \theta^{\alpha}}+D \frac{\partial \mathcal{L}_{e}}{\partial \Theta^{\alpha}}\right) \\
& +\delta \omega^{\alpha \beta} \wedge\left[D \frac{\partial \mathcal{L}_{e}}{\partial \Omega^{\alpha \beta}}-\frac{1}{2}\left(\theta_{\alpha} \wedge \frac{\partial \mathcal{L}_{e}}{\partial \Theta_{\beta}}-\theta_{\beta} \wedge \frac{\partial \mathcal{L}_{e}}{\partial \Theta_{\alpha}}\right)\right] \\
& +\delta \lambda^{\alpha} \wedge \frac{\partial \mathcal{L}_{e}}{\partial \lambda^{\alpha}}+d\left(\delta \theta_{\alpha} \wedge \frac{\partial \mathcal{L}_{e}}{\partial \Theta^{\beta}}+\delta \omega^{\alpha \beta} \wedge \frac{\partial \mathcal{L}_{e}}{\partial \Omega^{\alpha \beta}}\right)
\end{aligned}
$$

The definition of the derivative of the Lagrangian volume 4 -form with respect to a $p$-form has been used with the usual partial derivative symbol as is customarily done [29, 34] in the literature. For the explicit expression for $\mathcal{L}_{e}$ in (4), after evaluating some of the derivatives, the general expression (6) then takes the form

$$
\begin{aligned}
\delta \mathcal{L}_{e} & =\delta \theta^{\alpha} \wedge * E_{\alpha} \\
& +\delta \omega_{\alpha \beta} \wedge\left[\Pi^{\alpha \beta}-\frac{1}{2}\left(\theta^{\alpha} \wedge \lambda^{\beta}-\theta^{\beta} \wedge \lambda^{\alpha}\right)\right] \\
& +\delta \lambda^{\alpha} \wedge \Theta_{\alpha}+d\left(\delta \theta_{\alpha} \wedge \lambda^{\alpha}+\delta \omega_{\alpha \beta} \wedge * X^{\alpha \beta}\right),
\end{aligned}
$$

where the following auxiliary tensor-valued forms are defined in terms of the derivatives that come out in the variational derivative with respect to gravitational variables

$$
\Pi^{\alpha \beta} \equiv D * X^{\alpha \beta} \equiv D \frac{\partial \mathcal{L}}{\partial \Omega^{\alpha \beta}} .
$$

Assuming that the variations of the variables vanish on the boundary, the field equations for the connection 1form then yields the algebraic relation

$$
\Pi^{\alpha \beta}=\frac{1}{2}\left(\theta^{\alpha} \wedge \lambda^{\beta}-\theta^{\beta} \wedge \lambda^{\alpha}\right) .
$$

The connection equations can be solved for the Lagrange multiplier form $\lambda^{\alpha}$. The Lagrange multiplier $(n-2)$-form $\lambda^{\alpha}$ is a vector-valued 2-form while $\Pi^{\alpha \beta}$ is tensor-valued 3form in four dimensions and they have an equal number of components [28]. The equivalence can uniquely be expressed by inverting (9) to write $\lambda_{\alpha}$ in terms of $\Pi^{\alpha \beta}$ as

$$
\lambda^{\beta}=2 i_{\alpha} \Pi^{\alpha \beta}-\frac{1}{2} \theta^{\beta} \wedge i_{\mu} i_{\nu} \Pi^{\mu \nu} .
$$

This formula can be obtained by calculating contractions of Eq. (9). The right-hand side is to be calculated subject to the vanishing torsion constraint $\Theta^{\alpha}=0$, which results from the variational derivative of the extended Lagrangian with respect to the Lagrange multiplier 2forms. As will be apparent with the explicit calculations 
in the subsequent sections, the auxiliary tensor-valued forms $X^{\alpha \beta}$ and $\Pi^{\alpha \beta}$ are also quietly suitable in the construction of a scalar-tensor equivalent Lagrangian for a given modified gravitational Lagrangian as well.

Finally, returning to the coframe equations, in the general case, the metric equations are derived from the coframe variational derivative in the form

$$
\frac{\delta \mathcal{L}_{e}}{\delta \theta^{\alpha}} \equiv * E^{\alpha}=\frac{\partial \mathcal{L}}{\partial \theta^{\alpha}}+D \lambda^{\alpha}=0 .
$$

The Lagrange multipliers $\lambda_{\alpha}$, which are to be calculated by using (10) for the vanishing torsion constraint, also contribute to the metric equations. The 1 -form $E_{\alpha}=$ $E_{\alpha \beta} \theta^{\beta}$ in the context of the discussion below, can be regarded as a generalization of the Einstein 1-form $G_{\alpha}=$ $G_{\alpha \beta} \theta^{\beta}$. The tensor $E_{\alpha \beta}$ is symmetric in its indices as a result of the local Lorentz invariance, that is, $\theta^{\beta} \wedge * E^{\alpha}-$ $\theta^{\alpha} \wedge * E^{\beta}=0$, and it is covariantly constant as a result of the general coordinate invariance of the Lagrangian, i. e., $D * E^{\alpha}=0$ as in the case of the Einstein-Hilbert action.

The first order formalism naturally allows one to consider the connection as an independent gravitational variable, as in the so-called Palatini approach, by dropping the constraint on the connection, i.e., by setting $\lambda_{\alpha}=0$. As a consequence, both the Palatini and the metric variational procedures yield the same metric equations only in the case $\lambda_{\alpha}=0$ [35]. The prime example of this exceptional case is the well-known Einstein-Hilbert action. The discussion of the explicit examples below will involve the pseudo-Riemannian case by constraining the connection to be Levi-Civita. The field equations derived from the Palatini variational method will also be considered for $f(R)$ and for some curvature-squared gravitational Lagrangian forms in connection with the construction of ST equivalence.

\section{ST EQUIVALENTS FOR $f(R)$ THEORIES}

In this section, in a streamlined fashion, the wellknown equivalence between $f(R)$ theories and ST theories is presented in the Riemannian case as well as the non-Riemannian geometries with either torsion or nonmetricity of $f(R)$ models relative to an orthonormal coframe with appropriate constraints. These $f(R)$ models and their ST equivalence have recently been studied in [30]. The use of constrained first order formalism facilitates the study of all these subtheories in a unified manner and ST equivalents can be introduced in a unified manner as well.

An additional advantage of the presentation in this framework, as indicated also in [23], is that it allows one to further generalize such an equivalence for modified gravitational actions involving more general curvature invariants at the level of the field equations. In the subsequent section, the results will be generalized to modified gravitational actions based on quadratic curvature scalars. The modified gravitational models will be considered without introducing any matter field coupling.

\section{A. $f(R)$ model in the Riemannian context}

It is well known that the field equations that follow from the modified gravitational action

$$
\mathcal{L}=f(R) * 1
$$

can be cast into equivalent Brans-Dicke-type scalartensor theory with a potential term. This is usually achieved by rewriting (12) as

$$
\mathcal{L}=\left[f(\chi)+f^{\prime}(\chi)(\chi-R)\right] * 1
$$

using an auxiliary field $\chi$ where the prime denotes differentiation with respect to $\chi$. Under the condition $f^{\prime \prime}(\chi) \neq 0$, the field redefinition $f(\chi)$ then immediately yields the scalar-tensor equivalent for (12) see, e.g., [1, 2].

However, in defining scalar-tensor equivalents below, a slightly different approach will be adopted by making use of the explicit expressions for the variational derivatives relative to an orthonormal coframe. Thus, in order to derive the ST equivalent to the Lagrangian (12), it is convenient to begin with the variational derivative of the Lagrangian density (12) without considering the constraint term. Later, in deriving the corresponding field equations, the Lagrangian will be extended by the constraint term $\mathcal{L}_{L M}=\lambda_{\alpha} \wedge \Theta^{\alpha}$. Explicitly, in the first order formalism, the variational derivative of (12) can be written in the form 31.

$$
\delta \mathcal{L}=(\delta f) * 1+f \delta * 1=f^{\prime} \delta(R * 1)+\left(f-R f^{\prime}\right) \delta * 1
$$

without the need for further evaluation of the variations $\delta(R * 1)$ and $\delta * 1$.

First, an important observation that later will be made essential use of is that the expression (14) for the variational derivative, in fact, holds for any scalar built out of other curvature scalars in the place of the simplest scalar $R$. Thus, (14) turns out to have enough generality that allows the extension of ST equivalence above.

Second, the coefficient of the variational derivative in the first term indicates that the function $f^{\prime} \equiv \frac{d f}{d R}$ acts like a nonminimally coupled scalar field similar to the BransDicke scalar field. The convenient field redefinitions that allow one to cast the theory into a ST-type theory can in fact be found, without carrying out the variational derivatives further. The expression of the second term on the right-hand side of (14) is particularly useful in this regard.

On the other hand, consider the following action

$$
\mathcal{L}_{S T}\left[\phi, \theta^{\alpha}, \Omega^{\alpha}{ }_{\beta}\right]=\phi R * 1-V(\phi) * 1,
$$

with a typical scalar field nonminimally coupled to gravity and having a potential term, which is to be made 
precise below, with no accompanying kinetic term in contrast to the standard Brans-Dicke type ST Lagrangian. Note at this point that, although the scalar field has no kinetic term, as will also be shown below, the metric field equations render $\phi$ a dynamical field as a consequence of the nonminimal coupling. The total variational derivative of (15) can easily be calculated to be

$$
\delta \mathcal{L}_{S T}=\phi \delta(R * 1)-V(\phi) \delta * 1+\delta \phi\left(R-\frac{d V}{d \phi}\right) * 1 .
$$

The first term on the right-hand side of (16) contributes to the coframe as well as to the connection equations whereas the second term contributes to the coframe equations. Moreover, at this stage in deriving an ST equivalent Lagrangian, the explicit form of these variational derivatives is not needed. Finally, the last term on the right-hand side of the variational derivative (16) yields the scalar field equation as

$$
R=\frac{d V}{d \phi}
$$

This constraint is identically satisfied if the potential $V(\phi)$ is chosen to be the Legendre transform of $f(R)$, that is,

$$
V(\phi) \equiv \phi R-f(R)
$$

where it is assumed that $f^{\prime \prime} \neq 0$ and thus the definition $\frac{d f}{d R}=f^{\prime}(R) \equiv \phi$ is invertible so that one at least locally has $R=R(\phi)$. Therefore, the potential $V(\phi)$ determined by the explicit form of the function $f(R)$ and the identification $f^{\prime} \equiv \phi$ brings the modified action (12) to its ST equivalent given in (15). Consequently, it is possible to formulate the field equations using either $\phi$ or $f^{\prime}$. Thus, the construction of the dynamical equivalent model amounts to employing Legendre transform of $f(R)$ with the scalar field $f^{\prime}=\phi$. The ST equivalence leads to second order equations in the metric. In addition, it introduces a nonminimally coupled scalar field which satisfies some second order equations.

Subsequently, one includes the constraint that the torsion vanishes by a Lagrange multiplier term $\mathcal{L}_{L M}=$ $\lambda_{\alpha} \wedge \Theta^{\alpha}$ giving the extended Lagrangian density

$$
\mathcal{L}_{e}\left[f^{\prime}, \theta^{\alpha}, \Omega^{\alpha}{ }_{\beta}, \Theta^{\alpha}, \lambda_{\alpha}\right] \equiv \mathcal{L}\left[f^{\prime}, \theta^{\alpha}, \Omega^{\alpha}{ }_{\beta}\right]+\mathcal{L}_{L M}
$$

and the metric equations then follow from the variational derivative of the extended Lagrangian with respect to coframe 1-forms. The total variational derivative is

$$
\begin{aligned}
\delta \mathcal{L}_{e} & =\delta \theta_{\alpha} \wedge\left(f^{\prime} \Omega_{\mu \nu} \wedge * \theta^{\alpha \mu \nu}+D \lambda^{\alpha}\right) \\
& +\delta \omega_{\alpha \beta} \wedge\left[D * f^{\prime} \theta^{\alpha \beta}-\frac{1}{2}\left(\theta^{\alpha} \wedge \lambda^{\beta}-\theta^{\beta} \wedge \lambda^{\alpha}\right)\right] \\
& +\delta \lambda_{\alpha} \wedge \Theta^{\alpha}+d\left(\delta \theta_{\alpha} \wedge \lambda^{\alpha}+\delta \omega_{\alpha \beta} \wedge * X^{\alpha \beta}\right)
\end{aligned}
$$

In the present case, the explicit form of the auxiliary forms are $X^{\alpha \beta}=f^{\prime} \theta^{\alpha \beta}$ and $\Pi^{\alpha \beta}=D * X^{\alpha \beta}$ which are to be calculated subject to the vanishing torsion constraint.
Subsequently, the resulting expression is used to express $\lambda^{\alpha}$ in terms of the gravitational variables with the help of (10). The metric field equations $\frac{1}{2} * E^{\alpha}=0$ that follow from coframe variation then take the form

$$
-f^{\prime} * G^{\alpha}+\frac{1}{2}\left(f-R f^{\prime}\right) * \theta^{\alpha}+D *\left(d f^{\prime} \wedge \theta^{\alpha}\right)=0 .
$$

Equivalently, in terms of the Legendre transform of $f(R)$, the field equations can be rewritten in terms of the scalar field $f^{\prime} \equiv \phi$ as

$$
-\phi * G^{\alpha}+D *\left(d \phi \wedge \theta^{\alpha}\right)-\frac{1}{2} V(\phi) * \theta^{\alpha}=0 .
$$

Although the field equations (22) have resemblance with the Brans-Dicke (BD) field equations (cf., for example, the form of the Brans-Dicke equations given in [36]) they do not involve the Brans-Dicke parameter $\omega$ as in the original BD Lagrangian. Such a theory (without the potential term for the scalar field) was introduced in connection with gravity theory with a Yukawa-type term in the Newtonian limit [37]. On the other hand, on the basis of ST equivalents of such theories, the value of the BD parameter $\omega=0$ implies that such theories are ruled out by solar system tests 38.

The dynamical equations for the scalar field can be found by tracing the metric equations and one obtains the equation

$$
\Delta \phi+U(\phi)=0
$$

where

$$
U(\phi) \equiv \frac{1}{(n-1)}\left[\phi \frac{d V}{d \phi}-(n-2) V(\phi)\right]
$$

is introduced and the Laplace-Beltrami operator $\Delta$ can be defined in terms of the exterior differential and the codifferential $* d^{\dagger}=(-1)^{p} d *$ as $\Delta=d^{\dagger} d+d d^{\dagger}$ acting on $p$-forms.

In the Riemannian case discussed in this subsection, the Riemannian connection is a quantity derived from the metric. On the other hand, the modified gravitational Lagrangians of $f(R)$ form are mathematically simple enough to accommodate independent connection with torsion and/or nonmetricity. In such models the torsion and/or nonmetricity induces minimal coupling terms for the scalar field in the scalar-tensor type gravity [39]. Consequently, the ST equivalents for the $f(R)$ models with torsion and/or nonmetricity are quite similar to the Riemannian case [11]. The ST equivalents for these models are studied in some detail in the next two subsections using the first order formalism formulas discussed above.

\section{B. Riemann-Cartan type $f(R)$ model}

Although both the Palatini and the metric variational approaches yield the same equations for the metric for the Einstein-Hilbert action, for the modified actions of the form $f(R) * 1$, they lead to distinct field equations. 
This subsection is devoted to the $f(R)$ model for which the connection is assumed to be metric compatible but have a nonvanishing torsion. The field equations can easily be obtained from those of the metric case. Explicitly, by setting $\lambda^{\alpha}=0$ in (20), $R$ is assumed to be scalar curvature corresponding to the non-Riemannian connection $\Gamma_{\beta}^{\alpha}$. The connection equations $\Pi^{\alpha \beta}=0$ can be written as

$$
D(\Gamma) * f^{\prime} \theta^{\alpha \beta}=d f^{\prime} \wedge * \theta^{\alpha \beta}+\Theta^{\mu} \wedge f^{\prime} * \theta_{\mu}^{\alpha \beta}=0,
$$

where $D(\Gamma)$ is now the covariant exterior derivative with respect to a connection form $\Gamma_{\beta}^{\alpha}$. (25) is an algebraic equation for torsion 2 -form and it can uniquely be solved for torsion as

$$
\Theta^{\mu}=-\frac{1}{n-2} \theta^{\mu} \wedge d \ln f^{\prime},
$$

where $n$ denotes the number of dimensions. Similarly, the coframe variations then take the form

$$
-f^{\prime} * G^{\alpha}(\Gamma)-\frac{1}{2}\left(R(\Gamma) f^{\prime}-f\right) * \theta^{\alpha}=0,
$$

where $* G^{\alpha}(\Gamma)$ is the Einstein form corresponding to the curvature of the connection $\Gamma$. It is convenient to work with the ST equivalent which can be introduced in the same way as before by defining $\phi \equiv f^{\prime}$ with the Legendre transformation $V(\phi) \equiv R(\Gamma) f^{\prime}-f$.

It is possible to rewrite (27) in terms of Riemannian quantities as follows. In order to do so one uses the well-known fact that a general connection $\Gamma_{\beta}^{\alpha}$ can be decomposed into the torsion and the nonmetricity parts in addition to a Riemannian part $\omega^{\alpha}{ }_{\beta}$ as

$$
\Gamma_{\beta}^{\alpha}=\omega_{\beta}^{\alpha}+K_{\beta}^{\alpha}-\left(i^{\alpha} Q_{\beta \mu}-i_{\beta} Q_{\mu}^{\alpha}\right) \theta^{\mu}+Q_{\beta}^{\alpha},
$$

where $K_{\beta}^{\alpha}$ is the contorsion 1-form defined in terms of the torsion 2-form as $\Theta^{\alpha}=K_{\beta}^{\alpha} \wedge \theta^{\beta}$ and $Q_{\alpha \beta}$ is the nonmetricity 1 -form defined by

$$
Q_{\alpha \beta}=-\frac{1}{2} D \eta_{\alpha \beta}=\frac{1}{2}\left(\Gamma_{\alpha \beta}+\Gamma_{\beta \alpha}\right),
$$

and $\omega_{\alpha \beta}$ is the Riemannian connection satisfying $\omega_{\alpha \beta}+$ $\omega_{\beta \alpha}=0$ and $d \theta^{\alpha}+\omega_{\beta}^{\alpha} \wedge \theta^{\beta}=0$ [28, 39]. Explicitly, in the particular case of a Riemann-Cartan type $f(R)$ model, by making use of (26) one finds that

$$
K_{\beta}^{\alpha}=-i^{\alpha}(d \ln \phi) \theta_{\beta}+i_{\beta}(d \ln \phi) \theta^{\alpha},
$$

and consequently, using this in (28), it is possible to decompose the connection form $\Gamma_{\beta}^{\alpha}$ into the Riemannian $\omega^{\alpha}{ }_{\beta}$ and non-Riemannian parts as

$$
\Gamma_{\beta}^{\alpha}=\omega_{\beta}^{\alpha}-i^{\alpha}(d \ln \phi) \theta_{\beta}+i_{\beta}(d \ln \phi) \theta^{\alpha} .
$$

On the other hand, note that (31) is precisely the conformal transformation law for the connection forms $\omega^{\alpha}{ }_{\beta}$ under the conformal transformations of the metric $g \mapsto$ $(\ln \phi)^{2} g$ in terms of the scalar field $\phi=f^{\prime}$. Consequently, in a Riemann-Cartan type $f(R)$ theory, which leads to an algebraic torsion, the decomposition (31) allows one to write (27) in terms of pseudo-Riemannian quantities and a scalar field as

$$
\begin{gathered}
-\phi * G^{\alpha}(\omega)+D(\omega) *\left(d \phi \wedge \theta^{\alpha}\right)-\frac{1}{2} V(\phi) * \theta^{\alpha} \\
+\left(\frac{n-1}{n-2}\right) \frac{1}{\phi} * T^{\alpha}[\phi]=0
\end{gathered}
$$

where all quantities are pseudo-Riemannian and $T^{\alpha}[\phi]$ is the energy-momentum 3 -form for the scalar field defined by

$$
* T^{\alpha}[\phi]=-\frac{1}{2}\left\{\left(i^{\alpha} d \phi\right) * d \phi+d \phi \wedge i^{\alpha} * d \phi\right\} .
$$

Consequently, compared to the equations (22) of the metric case, the additional assumption of a nonvanishing torsion further induces scalar field gravitational couplings reflected by the last term in (27). In contrast to the Einstein-Hilbert action, for the general case $f(R) \neq R$, the Palatini variational procedure yields metric equations that are not equivalent to those obtained by the metric variational procedure by construction [1, 2]. The geodesics defined by $\Gamma_{\beta}^{\alpha}$ will clearly be different from those of $\omega_{\beta}^{\alpha}$.

\section{C. $f(R)$ model with nonmetricity}

$f(R)$ model are simple yet rich enough to allow independent connection $\Gamma$ to have nonmetricity but vanishing torsion as well. This subcase can be obtained from the Riemannian case by simply assuming an algebraic nonmetricity constraint while keeping the zero torsion constraint. In this case one has to pay attention to the fact that the raising and lowering of the indices with $\eta^{\alpha \beta}$ and $\eta_{\alpha \beta}$ do not commute with covariant exterior derivative $D(\Gamma)$.

Explicitly, the coframe equations have formally the same as those of the Riemannian case, which takes the form

$$
-f^{\prime} * G^{\alpha}(\Gamma)-\frac{1}{2}\left(R(\Gamma) f^{\prime}-f\right) * \theta^{\alpha}+D(\Gamma) \lambda^{\alpha}=0,
$$

where the Einstein form $* G^{\alpha}(\Gamma)$ and the scalar curvature $R(\Gamma)$ are to be now computed from curvature of the connection $\Gamma_{\beta}^{\alpha}$ with nonmetricity. The equations for the connection 1-form $\delta \mathcal{L} / \delta \Gamma^{\alpha}{ }_{\beta}=0$ accordingly take the form

$$
D(\Gamma)\left[f^{\prime} *\left(\theta_{\alpha} \wedge \theta^{\beta}\right)\right]-\lambda_{\alpha} \wedge \theta^{\beta}=0,
$$

subject to the vanishing torsion constraint $\Theta^{\alpha}=0$. These equations can explicitly be written as

$$
\left(d f^{\prime} \eta_{\alpha \mu}-\frac{1}{2} f^{\prime} Q_{\alpha \mu}\right) \wedge * \theta^{\mu \beta}+f^{\prime} \eta_{\alpha \mu} \Theta_{\nu} \wedge * \theta^{\alpha \beta \nu}-\theta_{\alpha} \wedge \lambda^{\beta}=0,
$$

in terms of nonmetricity and torsion forms. The connection equations in this case can be regarded as an equation for both nonmetricity 1-form and the Lagrange multiplier 
2 -form. The second term on the left-hand side is to be dropped because of the constraint $\Theta^{\alpha}=0$. (36) then admits the following simple solution for nonmetricity 1form and the Lagrange multiplier 2-form. If one assumes that

$$
\eta_{\alpha \mu} d f^{\prime}-\frac{1}{2} f^{\prime} Q_{\alpha \mu}=0,
$$

is satisfied by the nonmetricity 1-form then one has $\lambda^{\beta}=$ 0 . The nonmetricity 1-form (37) has the same diagonal elements and therefore is proportional to the Weyl 1-form defined as the trace part of a general nonmetricity 1-form [28].

As in the Riemann-Cartan subcase, the connection $\Gamma_{\beta}^{\alpha}$ having nonmetricity can be decomposed into Riemannian in addition to non-Riemannian parts as

$$
\Gamma_{\beta}^{\alpha}=\omega_{\beta}^{\alpha}-2 i^{\alpha}\left(d \ln f^{\prime}\right) \theta_{\beta}+2 i_{\beta}\left(d \ln f^{\prime}\right) \theta^{\alpha}+2 \delta_{\beta}^{\alpha} d \ln f^{\prime} .
$$

Thus, the $f(R)$ model also allows a connection with nonmetricity determined by the gradient of the scalar $d f^{\prime}$. The decomposition (38) also allows one to write the coframe equations (34) in terms of Riemannian quantities corresponding to $\omega_{\beta}^{\alpha}$ and the scalar field $\phi \equiv f^{\prime}$. One finds that (34) can be rewritten as

$$
\begin{aligned}
& -\phi * G^{\alpha}(\omega)+\left(\frac{n}{2}-1\right) D(\omega) *\left(d \phi \wedge \theta^{\alpha}\right)-\frac{1}{2} V(\phi) * \theta^{\alpha} \\
& +\frac{(2-n)(n-1)}{2 \phi}\left[\left(i^{\mu} d \phi\right) \wedge * d \phi-\frac{n-7}{n-1} d \phi \wedge i^{\mu} * d \phi\right] \\
& =0 .
\end{aligned}
$$

As in the case of the Riemann-Cartan type $f(R)$ model, the nonmetricity gradient introduces an additional gravitational coupling term of scalar field to gravity. However, the induced scalar coupling terms explicitly depend on the number of the dimensions and in particular for the number of dimension $n=4$, the expression reduces to

$$
-\phi * G^{\alpha}(\omega)+D(\omega) *\left(d \phi \wedge \theta^{\alpha}\right)-\frac{1}{2} V(\phi) * \theta^{\alpha}+\frac{3}{\phi} * T^{\alpha}[\phi]=0
$$

where $* T^{\alpha}[\phi]$ is defined as in (33).

It is interesting to note that it is possible to constrain nonmetricity form by introducing the constraint term

$$
\rho_{\alpha}^{\beta} \wedge\left(Q_{\beta}^{\alpha}-\delta_{\beta}^{\alpha} d h(R)\right),
$$

where $h(R)$ is a given function of the scalar curvature and $\rho^{\beta}{ }_{\alpha}$ is symmetric Lagrange multiplier $(n-1)$-form. The variational derivative of the $f(R)$ model with the constraint (41) is, however, technically more involved than the case discussed. At this point, it is convenient to note that the constrained first order formalism encompasses the recent $\mathrm{C}$-theories which extrapolate and interpolate the metric and the Palatini methods [40]. In the general C-theory framework, independent connection has nonmetricity characterized by a (co)vector and it is derived from a metric conformally related to the independent metric depending only on the scalar curvature of the connection. On the other hand, in the first order formalism one proceeds in the opposite direction starting with a prescribed nonmetricity on independent connection [41].

The two simple non-Riemannian cases discussed above therefore imply that a general $f(R)$ model can be formulated in the framework of constrained first order formalism to have prescribed torsion and/or nonmetricity and these non-Riemannian models can be cast in a Riemannian form with the non-Riemannian quantities inducing further gravitational couplings of the scalar fields in the corresponding ST equivalents.

With regard to the ST equivalence, the generalization of the above discussion to the case with general curvature invariant in the place of the scalar curvature $R$ will explicitly studied below. The connection equations for generic $f(R)$ models be involve the term $\partial \mathcal{L} / \partial \Omega_{\alpha \beta}=f^{\prime} * \theta^{\alpha \beta}$ and lead to an algebraic equation for torsion and therefore the torsion does not propagate. On the other hand, the connection equations are more complicated since $\partial \mathcal{L} / \partial \Omega_{\alpha \beta}=f^{\prime} * \theta^{\alpha \beta}$ is more complicated than those of corresponding $f(R)$ models discussed above. Therefore, it is technically more difficult to treat more complicated gravitational Lagrangians in metric and Palatini formulations within the same framework and the corresponding ST equivalents in these distinct cases will have quite distinct features as well.

In the next section, a general form of the field equations for a modified Lagrangian based on an arbitrary curvature invariant and the construction of an ST equivalent is presented and the dynamical degree of freedom carried by the resulting scalar field is scrutinized.

\section{GENERAL SCALAR-TENSOR EQUIVALENCE FOR AN ARBITRARY CURVATURE INVARIANT}

A salient feature of the above discussion is that the study of the field equations for $f(R)$ theory can be carried out using an arbitrary scalar built out of the curvature tensor, say $P$. The above analysis can be generalized to relate the equations of motion for $\mathcal{L}_{P}=P * 1$, which can be written in the above notation as $* E_{P}^{\alpha}=0$, to those of $\mathcal{L}_{\text {mod.P }}=f(P) * 1$ without specifying the explicit form of the scalar $P$, except it is assumed that it is constructed out of an arbitrary contraction of Riemann tensor. It is also not necessary to give the explicit form of the field equations derived from the Lagrangian form $P * 1$.

Consider, now the variational derivative of $\mathcal{L}_{\text {mod.P }}$ for a given function $f(P)$. As in the $f(R)$ case, it is convenient to put it into the following preliminary form

$$
\delta \mathcal{L}_{\text {mod.P }}=f^{\prime} \delta \mathcal{L}_{P}-\delta \theta^{\alpha} \wedge\left(P f^{\prime}-f(P)\right) * \theta^{\alpha} .
$$

Now, one can make use of the general expression (6) for $\delta \mathcal{L}_{P}$ in (42) to obtain the variational derivative of the 
extended Lagrangian $\mathcal{L}_{\text {mod.P }}+\mathcal{L}_{L M}$ in the form

$$
\begin{aligned}
& \delta\left(\mathcal{L}_{\text {mod.P }}+\mathcal{L}_{L M}\right) \\
& =\delta \theta_{\alpha} \wedge\left[f^{\prime} \frac{\partial \mathcal{L}_{P}}{\partial \theta_{\alpha}}+D \lambda^{\alpha}+\left(f-P f^{\prime}\right) * \theta^{\alpha}\right] \\
& +\delta \omega_{\alpha \beta} \wedge\left[D\left(f^{\prime} \frac{\partial \mathcal{L}_{P}}{\partial \Omega^{\alpha \beta}}\right)-\frac{1}{2}\left(\theta^{\alpha} \wedge \lambda^{\beta}-\theta^{\beta} \wedge \lambda^{\alpha}\right)\right] \\
& +\delta \lambda_{\alpha} \wedge \Theta^{\alpha}+d\left(\delta \theta_{\alpha} \wedge \lambda^{\alpha}+\delta \omega_{\alpha \beta} \wedge f^{\prime} \frac{\partial \mathcal{L}_{P}}{\partial \Omega^{\alpha \beta}}\right) .
\end{aligned}
$$

This result expresses the relation between the auxiliary forms corresponding to the modified Lagrangian $\mathcal{L}_{\text {mod.P }}$ and the original Lagrangian $\mathcal{L}_{P}$ for the partial derivatives with respect to the gravitational variables. By considering (8), it is convenient to define the auxiliary tensor valued forms as

$$
* X_{m}^{\alpha \beta}=f^{\prime} * X_{P}^{\alpha \beta},
$$

and

$$
\Pi_{m}^{\alpha \beta}=D * X_{m}^{\alpha \beta}=f^{\prime} \Pi_{P}^{\alpha \beta}+\tilde{\Pi}^{\alpha \beta},
$$

in terms of the auxiliary forms $X_{P}^{\alpha \beta}$ and $\Pi_{P}^{\alpha \beta}$ of the $\mathcal{L}_{P}$. In Eq. (45), for convenience, $\tilde{\Pi}^{\alpha \beta}$ is defined to be

$$
\tilde{\Pi}^{\alpha \beta} \equiv d f^{\prime} \wedge * X_{P}^{\alpha \beta} .
$$

These definitions then lead to the following convenient split of the Lagrange multiplier form:

$$
\lambda^{\alpha}=f^{\prime} \lambda_{P}^{\alpha}+\tilde{\lambda}^{\alpha},
$$

with the second term is given by

$$
\tilde{\lambda}^{\alpha}=2 i_{\beta} \tilde{\Pi}^{\beta \alpha}+\frac{1}{2} \theta^{\alpha} \wedge i_{\mu} i_{\nu} \tilde{\Pi}^{\mu \nu} .
$$

Finally, the coframe equations for the modified Lagrangian $f(P) * 1$ then take the following general form

$$
f^{\prime} * E_{P}^{\alpha}-\left(P f^{\prime}-f(P)\right) * \theta^{\alpha}+d f^{\prime} \wedge \lambda_{P}^{\alpha}+D \tilde{\lambda}^{\alpha}=0 .
$$

This equation can be considered as a slightly generalized form of the metric $f(R)$ equations (21). If one takes $P=R$ in the above formulas, one then has $\lambda_{R}^{\alpha}=0$ identically with $\tilde{\lambda}^{\alpha}=4 *\left(d f^{\prime} \wedge \theta^{\alpha}\right)$ and consequently (49) reduces to (21).

An equation for the scalar $f^{\prime}$, as in the $f(R)$ case, can be derived by tracing the metric equations. The trace can explicitly be written as

$$
-2 d i_{\beta}\left(d f^{\prime} \wedge * i_{\alpha} X_{P}^{\alpha \beta}\right)-d f^{\prime} \wedge \theta_{\alpha} \wedge \lambda_{P}^{\alpha}+\left[4 f+(E-4 P) f^{\prime}\right] * 1=0
$$

where $E \equiv E^{\alpha}{ }_{\alpha}$ and $\lambda_{P}^{\alpha}$ is the Lagrange multiplier of the Lagrangian form $P * 1$. Thus, the second term contains only the first order derivatives of $f^{\prime}$ whereas the first term contains the second order derivatives of $f^{\prime}$. Recalling the definition of the auxiliary form (8), (50) implies that if the Lagrangian form $\mathcal{L}_{P}=P * 1$ is such that

$$
* i_{\alpha} X_{P}^{\alpha \beta}=-\theta_{\alpha} \wedge \frac{\partial \mathcal{L}_{P}}{\partial \Omega_{\alpha \beta}}=0
$$

then the equations for the scalar $f^{\prime}$ reduces to the algebraic equation $4 f+(E-4 P) f^{\prime}=0$. Note here that the second term in (50) also involves $i_{\alpha} X_{P}^{\alpha \beta}$ since

$$
\theta_{\alpha} \wedge \lambda_{P}^{\alpha}=2 i_{\beta} D *\left(i_{\alpha} X_{P}^{\alpha \beta}\right) .
$$

There are well-known cases where (152) is satisfied identically. Consider the gravitational Lagrangians involving only the quadratic curvature expressions, where $P$ is then quadratic in curvature components. In four dimensions, there is only one Lagrangian form for which (52) vanishes identically

$$
\lambda_{P}^{\alpha}=2 * C^{\alpha},
$$

where $* C^{\alpha}$ is the Cotton 2-form defined by $C^{\alpha} \equiv D\left(R^{\alpha}-\right.$ $\left.\frac{1}{6} R \theta^{\alpha}\right)$ in four dimensions [42]. In this case, it is easy to deduce that the corresponding quadratic curvature (QC) Lagrangian is then of the form $\mathcal{L}_{P}=P * 1=$ $R^{\alpha} \wedge * R_{\alpha}-\frac{1}{3} R^{2} * 1$, and is equivalent to a Weyl-squared Lagrangian up to a boundary term. This case will be discussed in a separate section below. For generic QC gravity, this conclusion can be extended to arbitrary dimensions simply by singling out the QC gravity Lagrangians whose field equations have second order trace. The QC Lagrangians having this particular property in arbitrary dimension $n \geq 3$ have been constructed in [32].

Any modified Lagrangian based on the general form $f\left(\right.$ Riemann $\left.^{2}\right)$ has an ST equivalent with dynamical scalar field. This follows from the fact that, with the definition $\Omega_{\alpha \beta} \wedge * \Omega^{\alpha \beta} \equiv K * 1$ for Lagrangian form $f(K) * 1$, the auxiliary form $X^{\alpha \beta}$ is given by $\frac{d f}{d K} * \Omega^{\alpha \beta}$ and its contraction does not vanish identically.

A trivial case for which the scalar field is nondynamical is the case where $\lambda_{P}^{\alpha}=0$ identically. In this case $\lambda_{P}^{\alpha}=0$ follows from $\Pi_{P}^{\alpha \beta}=D * X_{P}^{\alpha \beta}=0$ and consequently the only nonvanishing contribution to the modified Lagrange multiplier form comes from $\tilde{\Pi}^{\alpha \beta}=d f^{\prime} \wedge * X^{\alpha \beta}$, which also renders $f^{\prime}$ non-dynamical. Recalling the expression of the auxiliary form $* X_{P}^{\alpha \beta}$ defined in (8), the vanishing of Lagrange multiplier in the metric case requires

$$
D * X_{P}^{\alpha \beta}=D \frac{\partial \mathcal{L}_{P}}{\partial \Omega_{\alpha \beta}}=0,
$$

subject to the condition $\Theta^{\alpha}=0$. This requirement is identically satisfied if the partial derivative in (54) is, for example, of the form $* \theta^{\alpha \beta}$ corresponding to EinsteinHilbert action. The next complicated example for (54) is satisfied when the partial derivative is of the form

$$
\frac{\partial \mathcal{L}_{P}}{\partial \Omega_{\alpha \beta}}=\Omega_{\mu \nu} \wedge * \theta^{\alpha \beta \mu \nu},
$$

for which $D * X_{P}^{\alpha \beta} \equiv 0$ as a consequence of the Bianchi identity, $D \Omega_{\mu \nu} \equiv 0$, in addition to the vanishing torsion constraint $D \theta^{\alpha}=0$. "Integrating" (55), one finds that $\mathcal{L}_{P}$ is the well-known Gauss-Bonnet Lagrangian density

$$
G * 1 \equiv \Omega_{\alpha \beta} \wedge \Omega_{\mu \nu} \wedge * \theta^{\alpha \beta \mu \nu} .
$$


Consequently, for a modified gravitational Lagrangian density of the form $f(G) * 1$, the scalar tensor equivalence yields only a superficial field redefinition for $f^{\prime}$, since there is no dynamical degree of freedom resulting from the function $f^{\prime}$.

The use of the first order formalism relative to an orthonormal coframe makes the construction of ST equivalents transparent for a given modified gravitational Lagrangian and allows one to write the field equations in a formally simplified form in the pseudo-Riemannian case. The new insight gained through the study of the Lagrange multiplier term of the modified Lagrangian in terms of the original Lagrangian is that the equation for the scalar field in a generic ST equivalent is determined by the properties of the field equations of the original Lagrangian and the dynamical status of the scalar field depends on the form of the original Lagrangian through the trace of the derivative $\partial \mathcal{L}_{P} / \partial \Omega_{\alpha \beta}$.

Although it is only technically more involved, it is straightforward to extend the above discussion to the case where $f$ depends on an arbitrary number of arguments. In particular, note that the form of the metric equations (49), independent of the explicit form of the scalar $P$, facilitates the field redefinition $f^{\prime}=\phi$ and thus it motivates a dynamically equivalent multi-scalar-tensor model for the modified gravitational Lagrangian densities of the form $\mathcal{L}=f(R, Q, P, K, \ldots) * 1$ where $Q, P, K, \ldots$ are distinct scalars in the components of the curvature tensor. In the general case, the scalar $f^{\prime}$ has nonminimal coupling and is dynamical. On the other hand, in the case where the scalar field is nondynamical, the Legendre transform of $f$ becomes proportional to the trace of the equations that follow from $P * 1$ and the field redefinition $f^{\prime} \equiv \phi$ becomes superfluous.

As will also be studied to some extent in the examples below, the number of scalar fields in the ST equivalents can be less than the number of scalars on which a general $f$ depends. This is, in fact related to the property of the Legendre transform with constraints and consequently the number of scalar field equations can be less then the number of independent arguments of $f$. The constraints in the present context though are not related to the evolution of the gravitational field variables [23].

In the next section, these considerations will be applied to the explicit cases where the scalars $Q, P, K$ are assumed to be some quadratic curvature scalars and to their corresponding ST equivalents, which are relevant to some popular cosmological models.

\section{MODIFIED QUADRATIC CURVATURE LAGRANGIANS}

In order to study the multi-ST equivalents for modified gravity models at the level of the field equations, it is first convenient to study the field equations that follow from quadratic curvature models. Eventually, the field equations for the modified models will be formulated in terms of quadratic curvature gravity equations with nonminimally coupled scalar fields.

In four dimensions, there are three independent quadratic curvature scalars, namely $R^{2} * 1, R_{\alpha} \wedge * R^{\alpha}$ and $\Omega_{\alpha \beta} \wedge * \Omega^{\alpha \beta}$. Therefore the most general gravitational action density involving quadratic invariants consists of linear combinations of these individual densities. For what follows it is convenient to introduce the scalars $Q, P, K$ by the following definitions

$$
\begin{aligned}
& \mathcal{L}_{Q}=Q * 1 \equiv R^{2} * 1, \\
& \mathcal{L}_{P}=P * 1 \equiv R^{\alpha} \wedge * R_{\alpha}, \\
& \mathcal{L}_{K}=K * 1 \equiv \Omega^{\alpha \beta} \wedge * \Omega_{\alpha \beta} .
\end{aligned}
$$

In this section ST equivalents for the Lagrangian density of the form $f(Q, P, K) * 1$ will explicitly be constructed and the corresponding field equations will be derived in some generality.

As a result of the well-known fact that, in four spacetime dimensions, the variational derivative of the GaussBonnet term vanishes [43], i.e., $\delta\left(\Omega_{\alpha \beta} \wedge \Omega_{\mu \nu} \wedge * \theta^{\alpha \beta \mu \nu}\right)=0$, only two of the three Lagrangian densities in (57) are functionally independent. However, intending the modification of the quadratic curvature Lagrangian densities in the $f(R)$ spirit, it is convenient to work with all three scalars. For further calculational details for the variational derivatives of the Lagrangians relative to an orthonormal coframe considered here, see [31 33].

Since the field equations for any of the Lagrangians in (57) can be written in the same particular form, it is convenient to define the following total Lagrangian density,

$$
\mathcal{L}_{q c}=a \mathcal{L}_{Q}+b \mathcal{L}_{P}+c \mathcal{L}_{K},
$$

where $a, b, c$ are arbitrary coupling constants and Latin subscripts $Q, P, K$, for example, indicate tensorial quantities belonging to the Lagrangians $\mathcal{L}_{Q}, \mathcal{L}_{P}, \mathcal{L}_{K}$ respectively. Note that the scalar fields in the equivalent Lagrangians are not dynamical for every combination of the QC coupling constants $a, b, c$. For (58), one has

$$
\frac{\partial \mathcal{L}_{q c}}{\partial \theta^{\mu}}=\Omega_{\alpha \beta} \wedge i^{\mu} * X_{t}^{\alpha \beta}-i^{\mu} \mathcal{L}_{q c} \equiv * T_{q c}^{\mu},
$$

where the auxiliary 2 -form is explicitly given by

$$
\begin{aligned}
X_{q c}^{\alpha \beta} & =a X_{Q}^{\alpha \beta}+b X_{P}^{\alpha \beta}+c X_{K}^{\alpha \beta} \\
& =2 a R \theta^{\alpha \beta}+b\left(\theta^{\alpha} \wedge R^{\beta}-\theta^{\beta} \wedge R^{\alpha}\right)+2 c \Omega^{\alpha \beta} .
\end{aligned}
$$

Note that (59) can be written in a linear sum of the form

$$
* T_{q c}^{\alpha}=a * T_{Q}^{\alpha}+b * T_{P}^{\alpha}+c * T_{K}^{\alpha},
$$

with the help of (60). The auxiliary 3-form $* T_{q c}^{\alpha}$ has a mathematical structure that is formally analogous to energy-momentum form of a generic minimally coupled matter 2 -form field $F=\frac{1}{2} F_{\alpha \beta} \theta^{\alpha \beta}$ expressed relative to an orthonormal coframe (see, e.g., 44]). It is, for example, traceless (only in four dimensions): $T_{q c \alpha}^{\alpha}=0$. Thus, only 
the fourth order terms, the Lagrange multiplier terms, contribute to the trace of the metric equations. $X_{q c}^{\alpha \beta}$ is used to calculate $\lambda_{q c}^{\alpha}$ making use of $\Pi_{q c}^{\alpha \beta}=D * X_{q c}^{\alpha \beta}$ and the Lagrange multiplier can similarly be written as the linear sum of multipliers for individual QC components as

$$
\begin{aligned}
\lambda_{q c}^{\alpha} & =a \lambda_{Q}^{\alpha}+b \lambda_{P}^{\alpha}+c \lambda_{K}^{\alpha} \\
& =4 a *\left(d R \wedge \theta^{\alpha}\right)+b * D\left(2 R^{\alpha}+R \theta^{\alpha}\right) \\
& +c\left(4 i_{\beta} D * \Omega^{\beta \alpha}+\theta^{\alpha} \wedge i_{\mu} i_{\nu} D * \Omega^{\mu \nu}\right) .
\end{aligned}
$$

Consequently, with the help of the general formulas provided above, the field equations for the general quadratic curvature Lagrangian (58) can be written in the concise form

$$
* E_{q c}^{\alpha}=D \lambda_{q c}^{\alpha}+* T_{q c}^{\alpha}=0
$$

In the general case corresponding to (58), the form of the field equations is dimension independent and the trace of the field equations (63) in $n \geq 3$ can be found as

$$
E_{q c} * 1=-[4 a(n-1)+n b+c] d * d R+(n-4) \mathcal{L}_{q c},
$$

where $E_{q c} * 1 \equiv \theta_{\alpha} \wedge * E_{q c}^{\alpha}$ as before and in the present work we confine the study to $n=4$ dimensions.

After the discussion of the $\mathrm{QC}$ field equations in an appropriate form, now it is convenient to consider the gravitational Lagrangians that depend on the quadratic curvature invariants $Q, P, K$ in the form

$$
\mathcal{L}_{\text {mod. }}=f(Q, P, K) * 1 .
$$

Before studying modified gravitational field equations for specified functions $f$, it is first convenient to derive the field equations in some generality. As before, it is convenient to write the total variational derivative of the
Lagrangian $\mathcal{L}_{\text {mod }}$. in the form

$$
\begin{aligned}
\delta \mathcal{L}_{\text {mod. }} & =f_{Q} \delta \mathcal{L}_{Q}+f_{P} \delta \mathcal{L}_{P}+f_{K} \delta \mathcal{L}_{K} \\
& +\left(f-Q f_{Q}-P f_{P}-K f_{K}\right) \delta * 1 .
\end{aligned}
$$

The explicit form of the last term on the right-hand side, in this case, is the Legendre transform of the function $f(Q, P, K)$. By introducing the scalar fields

$$
\phi_{1} \equiv f_{Q}=\frac{\partial f}{\partial Q}, \phi_{2} \equiv f_{P}=\frac{\partial f}{\partial P}, \phi_{3} \equiv f_{K}=\frac{\partial f}{\partial K},
$$

the variational derivative of the modified Lagrangian $\mathcal{L}_{m}$ becomes equivalent to that of the multi-scalar-tensortype Lagrangian

$$
\mathcal{L}_{S T}=\phi_{1} \mathcal{L}_{Q}+\phi_{2} \mathcal{L}_{P}+\phi_{3} \mathcal{L}_{K}-V\left(\phi_{1}, \phi_{2}, \phi_{3}\right) * 1,
$$

provided that the potential term $V\left(\phi_{1}, \phi_{2}, \phi_{3}\right)$ for the scalar fields is taken to be the Legendre transform of $f(Q, P, K)$, namely

$V\left(\phi_{1}, \phi_{2}, \phi_{3}\right) \equiv Q f_{Q}+P f_{P}+K f_{K}-f(Q, P, K) . \quad(69)$ Note that the labels $Q, P, K$ stand for derivative only when they are written as a subscript of the function $f$ and in all other cases it is a discriminating label for the corresponding QC quantities.

Similar to the $f(R)$ theory case, the dependence of the multivariable function $f$ on a particular scalar introduces a corresponding scalar field by means of the Legendre transform. The number of scalar fields $\phi_{k}$ does in fact depend on the explicit form of the function $f$ and is equal to the rank of the Hessian matrix related to the Legendre transform of $f$. It is assumed that the function $f$ satisfies some regularity condition which ensures that the Legendre transformation is invertible. The Hessian matrix of the Legendre transformation $\left\{f_{P}, f_{Q}, f_{K}\right\} \mapsto\left\{\phi_{1}, \phi_{2}, \phi_{3}\right\}$ is assumed to be of rank three [23]. For the explicit examples discussed below, the relevant Hessian matrices are of lower rank and consequently the general formulas for multi-ST equivalence simplify considerably.

In terms of the new field variables defined above, the equations for the modified gravitational Lagrangian can be written concisely in the form

$$
\begin{aligned}
& \phi_{1} * E_{Q}^{\alpha}+\phi_{2} * E_{P}^{\alpha}+\phi_{3} * E_{K}^{\alpha}-V\left(\phi_{1}, \phi_{2}, \phi_{3}\right) * \theta^{\alpha}+2 d \phi_{1} \wedge i_{\beta} D * X_{Q}^{\alpha \beta}+2 d \phi_{2} \wedge i_{\beta} D * X_{P}^{\alpha \beta} \\
& +2 d \phi_{3} \wedge i_{\beta} D * X_{K}^{\alpha \beta}+2 D\left[\left(i_{\beta} d \phi_{1}\right) * X_{Q}^{\alpha \beta}+\left(i_{\beta} d \phi_{2}\right) * X_{P}^{\alpha \beta}+\left(i_{\beta} d \phi_{3}\right) * X_{K}^{\alpha \beta}\right]=0,
\end{aligned}
$$

relative to an orthonormal coframe [32]. Equations (70) can be considered to be an extension of the field equations (22). These metric equations follow from their multi-ST equivalent actions (68) which are called "dual" to the original modified actions 23].

For generic quadratic curvature gravity, the tensor- valued auxiliary forms $X_{q c}^{\alpha \beta}$ above involve the terms that are linear in the contraction of the curvature 2-form, Ricci 1-form and scalar curvature and consequently, the terms containing $X^{\alpha \beta}$ in (70) can be regarded as the interaction terms of the scalar fields $\phi_{k}$ with curvature for $k=1,2,3$. These terms generalize the second term in 
(21) in the sense that the expression $f^{\prime} \theta^{\alpha \beta}$ for the $f(R)$ model is replaced with the above $X^{\alpha \beta}$ 's that are linear in curvature components. The equations for the scalar fields then can be obtained by tracing the field equations as in the $f(R)$ case. In particular, tracing the general equation (70), it is easy to find that the scalar fields become nondynamical if the corresponding $X^{\alpha \beta}$ satisfies $i_{\alpha} X^{\alpha \beta}=0$. This important subcase will be studied in one of the case studies below. However, note that, in contrast to the simpler $f(R)$ case, the metric equations for the ST equivalent model are still fourth order in metric components in this case as well.

The field equation (70) encompasses all the cases below except for the sixth order case where the generalization of $d R \wedge * d R \equiv S * 1$ in the form $f(S)$ will also be considered. However, because the derivation of the field equations proceeds in a slightly different way than other quadratic curvature Lagrangians, it will be treated separately below. However, it is easy to include the scalar $S$ in the above framework once the corresponding field equations are obtained. Various examples that are of interest in applications of modified gravity ranging from cosmology to the topics relevant to the quantum properties of black holes are studied below.

\section{A. Modified Ricci-squared Lagrangian}

For a relatively simple application of the use of the general result obtained in the previous section, consider the case where $f=f(P)$ where $P$ is defined in (57). By making use of (70), the field equations for the Lagrangian density $f(P) * 1$ can immediately be written down as

$$
\begin{array}{r}
\phi * E_{P}^{\alpha}-V(\phi) * \theta^{\alpha}+2 d \phi \wedge i_{\beta} D * X_{P}^{\alpha \beta} \\
+2 D\left[(d \phi)_{\beta} * X_{P}^{\alpha \beta}\right]=0
\end{array}
$$

where in this case, $\phi \equiv \frac{d f}{d P}$, the potential is given by $V(\phi)=P \frac{d f}{d P}-f(P)$ and the explicit form of the equations for the Ricci-squared Lagrangian density $P * 1$ takes the form

$$
\begin{aligned}
* E_{P}^{\mu}=D * D\left(2 R^{\mu}+\right. & \left.R \theta^{\mu}\right)-\frac{1}{2}\left(i^{\mu} \Omega_{\alpha \beta}\right) \wedge * X_{P}^{\alpha \beta} \\
+ & \frac{1}{2} \Omega_{\alpha \beta} \wedge i^{\mu} * X_{P}^{\alpha \beta}=0
\end{aligned}
$$

with $X_{P}^{\alpha \beta}=\theta^{\alpha} \wedge R^{\beta}-\theta^{\beta} \wedge R^{\alpha}$. Inserting the expression (72) into (71) and then calculating the trace, after some algebra, one obtains

$$
\begin{gathered}
\phi d * d R-4 V(\phi) * 1+2 d \phi \wedge * d R \\
\quad+2 d\left[(d \phi)_{\alpha} * R^{\alpha}\right]+R d * d \phi=0 .
\end{gathered}
$$

The importance of the result (71) becomes more pronounced when it is considered in connection with the result that the modified gravitational Lagrangian based on $f(R, Q, P)=R+a Q+b P$ is equivalent to Einstein gravity interacting with additional fields [16, 45].
The field equations for the $f(P)$ Lagrangian in the first order formalism can easily be found simply by disregarding the term imposing the constraint $\Theta^{\alpha}=0$. In this case, the independent (metric compatible) connection and coframe equations respectively take the form

$$
\begin{aligned}
D(\Gamma) * f^{\prime} X_{P}^{\alpha \beta} & =0, \\
f^{\prime} * T_{P}^{\alpha}\left[\Omega_{\mu \nu}(\Gamma), X_{P}^{\mu \nu}\right]+\left(f-P f^{\prime}\right) * \theta^{\alpha} & =0,
\end{aligned}
$$

where $\Omega_{\mu \nu}(\Gamma)$ is the curvature 2-form corresponding to the connection $\Gamma_{\alpha \beta}$ and $D(\Gamma)$ stands for the covariant exterior derivative corresponding to the connection $\Gamma_{\alpha \beta}$. In the case $f(P)=P$, assuming that the connection $\Gamma_{\alpha \beta}$ is torsion free, for an Einstein manifold with the Ricci 1-form of the form $R^{\alpha}=k \theta^{\alpha}$ for some nonzero constant $k$, the connection equations (74) are satisfied identically since $X_{P}^{\alpha \beta}$ simplifies to $X_{P}^{\alpha \beta}=2 k \theta^{\alpha \beta}$. Moreover, the auxiliary 3 -form $* T_{P}^{\alpha}\left[\Omega_{\mu \nu}(\Gamma), X_{P}^{\mu \nu}\right]$, in this case takes the form

$$
* T_{P}^{\alpha}\left[\Omega_{\mu \nu}(\Gamma), X_{P}^{\mu \nu}\right]=k \Omega_{\mu \nu}(\Gamma) \wedge * \theta^{\alpha \mu \nu}-4 k^{2} * \theta^{\alpha},
$$

and consequently, the coframe equations (75) become the familiar vacuum Einstein field equations with a cosmological constant

$$
-2 f^{\prime} k * G^{\alpha}-f^{\prime} 4 k^{2} * \theta^{\alpha}+\left(f-4 k^{2} f^{\prime}\right) * \theta^{\alpha}=0,
$$

where $P=2 k^{2}$ for which $f(P)$ and $f^{\prime}(P)$ are also constants. These equations then constitute a certain part of the theorem on the so-called universal property of the Lagrangians depending on the Ricci-squared scalar 46]. Note however that in a metric theory, the generic quadratic curvature Lagrangians (as well as their modification in the $f(P)$ fashion) lead to fourth order equations, and they admit Einstein metric solutions for which the fourth order term $\left(D \lambda^{\alpha}\right.$ term) vanishes identically making the corresponding second order metric equations become identical to (75). One thus concludes that quadratic curvature models of type $f(P)$ in both first order and metric theories admit a common set of Einstein metric solutions. However, the number of dimensions is an important parameter, since for example, in three dimensions any Einstein metric has constant curvature. Moreover, $* T_{P}^{\alpha}$ has vanishing trace only in four dimensions and otherwise its trace is proportional to the density $\mathcal{L}_{P}$.

In the first order formalism and in the context of ST equivalence, the trace of the coframe equations yield $f-P f^{\prime}=0$ and thus the consistency of the equations requires the Legendre transform of $f(P)$ to vanish and they do have a scalar-tensor equivalent with a dynamical scalar field as in the case of the metric framework.

It is easy to show that the universality property of the quadratic curvature Lagrangian $f(P) * 1$ is also shared by $f(Q) * 1$ [47]. Take, for simplicity of the argument, the Lagrangian $Q * 1$. In the first order formalism and with the assumption $R^{\alpha}=k \theta^{\alpha}$, the corresponding auxiliary 2 -form then becomes $X_{Q}^{\alpha \beta}=8 k^{2} \theta^{\alpha \beta}$ and both the 
connection and the coframe equations are satisfied identically for a Riemannian connection. Consequently, the universality property of the $f(P) * 1$ in fact can be extended to the Lagrangian densities of the form $f(Q, P) * 1$.

For the case $f$ is a nonlinear function of $P$, the connection equations allow an algebraic torsion, cf. Eqn. (25), as in the case of Einstein-Cartan type $f(R)$ theory discussed in Sec. III.

Consequently, the connection $\Gamma_{\alpha \beta}$ has exactly the same decomposition as (31) with $f^{\prime}=f_{P}$ and the coframe equations assume the form

$$
\Omega_{\mu \nu}(\Gamma) \wedge i^{\alpha} * X^{\mu \nu}-\frac{1}{2} i^{\alpha} \mathcal{L}_{Q}=0
$$

which can be written in terms of the geometrical quantities belonging to the metric compatible and torsion-free connection $\omega$ related to $\Gamma$ by (26).

As in the case of the $R^{2}$ Lagrangian, the conformal transformation of the Ricci-squared Lagrangian and the corresponding field equations do not lead to a simplified form for ST equivalents. Because the expressions involved are relatively more manageable, it is preferable to work at the Lagrangian level to find how Ricci-squared Lagrangian transforms under conformal transformations.

Under the conformal transformation defined by $g \mapsto$ $\tilde{g}=\phi^{-2} g$ or equivalently, $\tilde{\theta}^{\alpha}=\phi^{-1} \theta^{\alpha}$ in terms of coframe basis 1-forms in $n$ dimensions, the transformation of the Ricci-squared Lagrangian density can be expressed in the form

$$
\begin{aligned}
\tilde{\mathcal{L}}_{P} & =\tilde{R}^{\mu} \wedge \tilde{*} \tilde{R}_{\mu}=\tilde{R}^{\mu \nu} \tilde{R}_{\mu \nu} \tilde{*} 1=\phi^{(n-4)}\left\{R^{\mu \nu} R_{\mu \nu}+2(2-n)\left[D_{\nu}(d \ln \phi)_{\mu}-(d \ln \phi)_{\nu}(d \ln \phi)_{\mu}\right] R^{\mu \nu}\right. \\
& +\left[(2-n)(d \ln \phi)_{\mu}(d \ln \phi)^{\mu}-D_{\mu}(d \ln \phi)^{\mu}\right] R+(2-n)^{2} D_{\mu}(d \ln \phi)^{\nu} D^{\mu}(d \ln \phi)_{\nu} \\
& -2(2-n)^{2} D^{\nu}(d \ln \phi)_{\mu}(d \ln \phi)^{\mu}(d \ln \phi)_{\nu}-(2-n)^{2}(n-1)\left[(d \ln \phi)_{\alpha}(d \ln \phi)^{\alpha}\right]^{2} \\
& \left.+(3 n-4)\left[D_{\mu}(d \ln \phi)^{\mu}\right]^{2}+2(2-n)(3-2 n) D^{\mu}(d \ln \phi)_{\mu}(d \ln \phi)_{\nu}(d \ln \phi)^{\nu}\right\} * 1
\end{aligned}
$$

where the shorthand notation $D_{\mu} \equiv i_{\mu} D$ has been used. Apparently, with a conformal transformation (79), the Ricci-squared Lagrangian $\mathcal{L}_{P}$ cannot be transformed into an Einstein-Hilbert Lagrangian with a nonminimally coupled scalar field in a simple manner as in $f(R)$ models [48].

\section{B. Modified quadratic curvature Lagrangians in a cosmological context}

In order to account for the late-time expansion rate of the Universe, the gravitational Lagrangians that modify the usual Einstein-Hilbert Lagrangian provide popular alternatives to the general relativistic cosmological models that involve dark energy and dark matter. Yet another approach is to alter the gravitational sector of the standard cosmological models so that it reconciles with the current cosmological observations. The ST equivalence for modified gravitational Lagrangians introduced above blurs the distinction between the two approaches. In this regard, it may be worthwhile to isolate the properties and predictions of some favorable modifications of the geometrical sector for a given cosmological model which cannot be reduced to simpler models having scalar degrees of freedom.

Although they are in conflict with solar system tests for gravity, the modified gravitational Lagrangians, for example, of the $f(R)$ form,

$$
f(R)=R-\frac{\mu^{4}}{R}
$$

are considered in attempts to account for the observed late-time cosmic acceleration. Here $\mu$ is a parameter which has the dimension of mass [49]. The basic idea behind the addition of $R^{-1}$ to the Einstein-Hilbert Lagrangian (80) is to change the field equations in the low curvature regime. Although it is possible to introduce the ST equivalent of (80) in the way highlighted in Sec. III which brings 80 to Einstein gravity with scalar field field coupled minimally to gravity (and nonminimally coupled to the matter fields if present), slightly more general modified gravitational Lagrangians are introduced later [25]. In a similar manner, in order to investigate the cosmological consequence of vacuum models by modifying the gravitational sector only, inverse powers of quadratic curvature invariants have been adopted. In particular, 80) is generalized to include the additional quadratic curvature scalars $Q, P, K$ in the form,

$$
f(R, Q, P, K)=R-\frac{\mu^{4 n+2}}{(a Q+b P+c K)^{n}},
$$

where $n$ is an integer. This form avoids the flat space solution and has desirable dynamical features similar to the Lagrangian in (80) in connection with the cosmic acceleration [25]. The ST equivalent Lagrangian for (81) has been introduced before in a different form in 22] in connection with its particle content when linearized around 
a curved background. It is argued that such modified gravitational Lagrangians are not conformally equivalent to Einstein gravity plus scalar matter sources [25] so that such models reflect solely the effects of modification of the gravitational sector. The multi-ST equivalent for (81), on the other hand, offers a somewhat simplified fourth order model with a nonminimally coupled single scalar degree of freedom.

For the sake of simplicity, here the modified Lagrangian density of the form

$$
\mathcal{L}_{\text {mod.qc }}=(a Q+b P+c K)^{m} * 1
$$

with $m$ is an integer will be considered. For this particular subclass of Lagrangians, the Legendre transform introduced above simplifies considerably to yield an explicit form of the potential term in terms of the scalar field. In this case, for the specific form of the function $f$ in (82), $f_{Q}, f_{P}$, and $f_{K}$ are all proportional to one another and thus it is possible to introduce a single scalar field coupled nonminimally to quadratic curvature Lagrangians. Explicitly, the ST equivalent for (82) becomes

$$
\mathcal{L}_{e q .}=\phi \mathcal{L}_{q c} * 1-V(\phi) * 1
$$

where, for convenience, the quadratic curvature Lagrangian $\mathcal{L}_{q c}$ is defined as

$$
\mathcal{L}_{q c} \equiv(a Q+b P+c K) * 1
$$

and the scalar field is obtained by the field redefinition $\phi \equiv(a Q+b P+c K)^{m-1}$. Consequently, the potential term in (83) takes the form of a fractional power law as

$$
V(\phi)=\frac{(m-1)}{m} \phi^{m /(m-1)}
$$

Therefore, the field equations for ST equivalent Lagrangian (83) can be derived from those of (84) by proceeding in the same way as in the first example above. The field equations for (84) can be written in the form

$$
* E_{q c}^{\alpha}=D \lambda_{q c}^{\alpha}+* T_{q c}^{\alpha}=0,
$$

where both terms are to be calculated by using the auxiliary tensor-valued 2-form

$$
X_{q c}^{\alpha \beta}=2 a R \theta^{\alpha \beta}+b\left(\theta^{\alpha} \wedge R^{\beta}-\theta^{\beta} \wedge R^{\alpha}\right)+2 c \Omega^{\alpha \beta} .
$$

Then the corresponding 2-form for (83) is $X_{e q .}^{\alpha \beta}=\phi X_{q c}^{\alpha \beta}$. Consequently, the Lagrange multiplier 2-forms for (83) and (84) are related by

$$
\lambda_{e q .}^{\alpha}=\phi \lambda_{q c}^{\alpha}+2(d \phi)_{\beta} * X_{q c}^{\alpha \beta} .
$$

Eventually, one finds the equation

$$
\begin{array}{r}
\phi * E_{q c}^{\alpha}+2 D\left[(d \phi)_{\beta} * X_{q c}^{\beta \alpha}\right]+2 d \phi \wedge i_{\beta} D * X_{q c}^{\beta \alpha} \\
+\frac{(1-m)}{m} \phi^{m /(m-1)} * \theta^{\alpha}=0
\end{array}
$$

which is a simplified and special case of the general expression (70). By tracing the field equations, after some algebra, one finds the equation for the scalar field

$$
\begin{array}{r}
(6 a+2 b) R d * d \phi+[4(6 a+2 b)+2 c] d \phi \wedge * d R \\
+(6 a+2 b+c) \phi d * d R+2(b+c) d *\left[(d \phi)_{\alpha} R^{\alpha}\right] \\
+\frac{4(1-m)}{m} \phi^{m /(m-1)} * 1=0 .
\end{array}
$$

Note that it is possible to recover the subcases mentioned above referring [32] with particular values of the coupling constants $a, b, c$ for which $\phi$ is nondynamical.

The subcase corresponding to $m=-1$ in (82) as well as the individual inverse powers of the quadratic curvature invariants supplemented with Einstein-Hilbert action have been studied in [25] in the context of cosmological models. In that work, it is stated that the generalization of the gravitational Lagrangian of the form (84) to the modified form (82) is not equivalent to Einstein gravity plus matter sources. What we see in this work is that any modification of the form $f(Q, P, K \ldots)$ induces scalar fields coupled nonminimally to individual curvature scalars $Q, P, K, \ldots$ in ST theory of the form (84) in the same sense that $f(R)$ models have ST equivalents. Thus, modifications of gravitational Lagrangian inevitably induce nonminimal scalar matter couplings and in this sense the ST equivalence blurs the distinction between adding matter fields, for example of the form dark energy/matter to Einstein equations and changing the gravitational sector starting from a modified gravitational action. In particular, in the case of the gravitational Lagrangian of the form (81), the scalar-tensor equivalent turns out to be a simpler gravitational model with a single scalar field coupled nonminimally to curvature components and having a suitable potential term for the resulting scalar field.

\section{Modified Weyl gravity}

For conformally invariant Weyl gravity [50, 51], the Lagrangian density can be written in terms of the contraction of the Weyl 2-form $C^{\alpha \beta}$ with itself in the following convenient form

$$
\begin{aligned}
\mathcal{L}_{W} & =C_{\alpha \beta} \wedge * C^{\alpha \beta} \\
& =\Omega_{\alpha \beta} \wedge * \Omega^{\alpha \beta}-\frac{1}{2} R_{\alpha} \wedge * R^{\alpha}+\frac{1}{6} R^{2} * 1,
\end{aligned}
$$

where Weyl 2-form $C^{\alpha \beta}$ is the trace-free part of the curvature 2 -form and can be expressed as

$$
C^{\alpha \beta}=\Omega^{\alpha \beta}-\frac{1}{2}\left(\theta^{\alpha} \wedge R^{\beta}-\theta^{\beta} \wedge R^{\alpha}\right)+\frac{1}{6} R \theta^{\alpha \beta} .
$$

With the help of the result that $\lambda_{W}^{\alpha}=2 C^{\alpha}$ where $C^{\alpha}=D L^{\alpha}$ is the Cotton 2-form derived from Schouten 1-form $L^{\alpha}=R^{\alpha}-\frac{1}{6} R \theta^{\alpha}$ [42] and using the above general formulas for quadratic curvature Lagrangians, it is possible to show that the field equations that follow from (91) take the form

$$
-R_{\beta} \wedge * C^{\alpha \beta}+D * C^{\alpha}+\frac{1}{2} * T_{W}^{\alpha}=0
$$


The last term explicitly has the form

$$
* T_{W}^{\mu} \equiv-\left(i^{\mu} C^{\alpha \beta}\right) \wedge * C_{\alpha \beta}+C^{\alpha \beta} \wedge i^{\mu} * C_{\alpha \beta},
$$

and vanishes identically in four dimensions [43]. In the present notation, this result can simply be obtained by making use of the identity $* C^{\alpha \beta}=\frac{1}{2} \epsilon^{\alpha \beta}{ }_{\mu \nu} C^{\mu \nu}$ satisfied by Weyl 2-form in four dimensions (the same identity is also used to simplify the Lagrange multiplier term as well, see [42]) in the expression (94). Consequently, the Weyl gravity equations further reduce to

$$
* E_{W}^{\alpha}=R_{\beta} \wedge * C^{\beta \alpha}+D * C^{\alpha}=0 .
$$

Note here that, in the component form the left hand side of (95) defines the Bach tensor $B_{\alpha \beta}$ [50]. In the present notation, and thus relative to an orthonormal coframe, it can be defined by introducing the vector-valued form $B^{\alpha}=E_{W}^{\alpha}$ by means of the identification $B^{\alpha}=B^{\alpha}{ }_{\beta} \theta^{\beta}$.

Now consider the field equations for the modified gravitational Lagrangian based on the Weyl scalar $C$ which can be defined as $C * 1 \equiv C_{\alpha \beta} \wedge * C^{\alpha \beta}$. In the spirit of the $f(R)$ model, consider the modified Lagrangian of the form

$$
\mathcal{L}_{\text {mod.W }}=f(C) * 1 .
$$

By making use of the field redefinitions $\frac{d f}{d C} \equiv \phi$, one has $\Pi_{\text {mod.W }}^{\alpha \beta}=2 \phi * C^{\alpha \beta}$ and therefore,

$$
\lambda_{W}^{\alpha}=2 \phi * C^{\alpha}+4(d \phi)_{\beta} * C^{\alpha \beta} .
$$

Consequently, the field equations take the form

$$
\phi * B^{\alpha}+2 d \phi \wedge i_{\beta} D * C^{\alpha \beta}+2 D\left[(d \phi)_{\beta} * C^{\alpha \beta}\right]-V(\phi) * \theta^{\alpha}=0,
$$

where the potential for the scalar is, as before, given by the Legendre transform $V(\phi)=f-C \phi$ with $\phi \equiv \frac{d f}{d C}$ and note also that $(d \phi)_{\beta}$ refers to the components of the 1 -form $d \phi$ relative to an orthonormal coframe. The field equations for Weyl theory (95) have vanishing trace, $B^{\alpha}{ }_{\alpha}=0$ as a consequence of trace-free property of Bach form which in turn follows from the common properties of Weyl 2-form and Cotton 2-form, that is $i_{\alpha} C^{\alpha \beta}=0$ and $i_{\alpha} C^{\alpha}=0$ respectively. This property is also maintained in the modified equations and thus leads to the fact that the trace of the vacuum field equations (98) implies the constraint

$$
f(C)-C f^{\prime}(C)=0,
$$

i.e., the potential for the scalar vanishes for consistency of the vacuum field equations. Thus, in this case the scalar field turns out to be nondynamical. Consequently, for the modified Weyl Lagrangian there is no advantage in field redefinitions via a Legendre transform. In terms of $f^{\prime}=$ $\frac{d f}{d C}$, the vacuum field equations (98) can be rewritten as

$$
f^{\prime} * B^{\alpha}+2 d f^{\prime} \wedge i_{\beta} D * C^{\alpha \beta}+2 D\left[\left(d f^{\prime}\right)_{\beta} * C^{\alpha \beta}\right]=0,
$$

subject to the condition (99). With regard to the practical use of the resulting field equations to the problems of interest in black hole physics for a given $f(C)$, (100) may, for example, allow one to adopt an alternative and direct approach to the analysis given in [52] provided that the above analysis is extended to higher dimensions.

\section{A MODIFIED SIXTH ORDER LAGRANGIAN}

The above procedure of introducing ST equivalents can also be extended to even higher order theories which was studied previously in 53. For example, gravitational Lagrangians involving cubic Ricci terms lead to sixth order metric equations. This section deals with another type of sixth-order gravitational Lagrangian based on the square of the gradients of the scalar curvature. In terms of differential forms such a Lagrangian form can be written as

$$
\mathcal{L}_{s}=d R \wedge * d R .
$$

For (101), one has $\Pi_{s}^{\alpha \beta}=D *\left[(\Delta R) \theta^{\alpha \beta}\right]$, and using the general formulas of Sec. 2 , one can easily show that it leads to the sixth order vacuum field equations

$$
* E_{s}^{\alpha}=-4(\Delta R) * R^{\alpha}+2 D *\left(\Delta d R \wedge \theta^{\alpha}\right)-* T_{s}^{\alpha}=0 .
$$

Equation (102) involves the Laplace-Beltrami operator $\Delta$ defined above and the Lagrange multiplier term, the second term in (102), contains sixth order partial derivatives of the metric components relative to a coordinate coframe. 3 -form $* T_{s}^{\alpha}$ is defined as

$$
* T_{s}^{\alpha} \equiv\left(i^{\alpha} d R\right) * d R+d R \wedge i^{\alpha} * d R,
$$

similar to the energy-momentum 3-form for the scalar field (33). Unlike the above quadratic curvature cases, 3form $* T_{s}^{\alpha}$ is not calculated by a corresponding auxiliary 2 -form $X^{\alpha \beta}$, but it results from the commutation of the variational derivative with $*$ for (101). However, eventually, the field equations written as (102) take a form similar to the those studied above in Sec. III. In fact, Eq. (102) can be brought into form more akin to those of a Brans-Dicke type ST theory as in the $f(R)$ case.

By comparing the field equation (102) with (22) it is possible to deduce that (102) can be written in the form analogous to (22) by the simple field redefinition $\Delta R \equiv \phi$. Note that such a mathematical connection is not obvious at the level of Lagrangian. In doing so, (102) then takes the form

$$
-4 \phi * R^{\alpha}-* T_{s}^{\alpha}+2 D *\left(d \phi \wedge \theta^{\alpha}\right)=0 .
$$

By taking the trace of Eq. (102), one finds

$$
E_{s \alpha}^{\alpha} * 1=3 \Delta \Delta R * 1+R \Delta R * 1-d R \wedge * d R=0,
$$

or equivalently, in terms of the scalar field it can be rewritten in the form

$$
3 d * d \phi+R \phi * 1-d R \wedge * d R=0 .
$$


The frame in (102) or (104) can be regarded as Jordan frame equations. One can try to put these into Einstein frame equations by considering a conformal transformation where equations coupled minimally to a scalar field with additional curvature-source terms.

The model based on $f(R) * 1+a d R \wedge * d R$ has been studied before in the context of cosmological perturbations [53, 54]. By making use of the field equations (21) and (102), it is possible to write the metric equations of such a Lagrangian in a form similar to (21), explicitly in the form

$$
-2 F * G^{\alpha}+2 D *\left(d F \wedge \theta^{\alpha}\right)+(f-R F) * \theta^{\alpha}-a * T_{s}^{\alpha}=0,
$$

where the definition $F \equiv f_{R}-2 a \Delta R$ has been introduced for convenience. In particular, for $f(R)=R+b Q$, it was shown that, by suitable field redefinitions after a conformal transformation analogous to the case for $f(R)$ models, it is possible to rewrite (107) as Einstein gravity with two interacting scalar fields [55].

It is now a convenient point to introduce ST equivalents for the generalization of the Lagrangian (102) in $f(R)$ fashion. One proceeds in the same way as in the examples before and first define the scalar $S$ via $S * 1 \equiv d R \wedge * d R$. Then, the formulas of previous sections can be used to derive the explicit form of the field equations based on the modified Lagrangian

$$
\mathcal{L}_{\text {mod.s }}=f(S) * 1,
$$

by making use of the field equations (102). Explicitly, by introducing $\frac{d f}{d S} \equiv f^{\prime} \equiv \phi$, they can be written in the form (70) as

$$
\begin{array}{r}
\phi * E_{s}^{\alpha}+V(\phi) * \theta^{\alpha}+2 \phi D *\left(\Delta d R \wedge \theta^{\alpha}\right) \\
+2 d \phi \wedge *\left(\Delta d R \wedge \theta^{\alpha}\right)+2 \Delta d R \wedge *\left(d \phi \wedge \theta^{\alpha}\right)=0,
\end{array}
$$

where the potential term is the Legendre transform $V(\phi) \equiv f-S f^{\prime}$. The trace of the modified field equations then yields a second order dynamical field equation for the scalar field coupled to the derivatives of the scalar curvature $R$ as

$6(\Delta \phi) \Delta R * 1-12 \Delta d R \wedge * d \phi+\phi E_{s \alpha}^{\alpha} * 1+4 V(\phi) * 1=0$.

Finally, it is a worthwhile to note that this example also illustrates a subtlety related to surface terms in the derivation of the field equations for modified gravity models. Recall that, using the properties of exterior derivative, coderivative and the Laplace-Beltrami operators, one has

$$
d(R * d R)=-R \Delta R * 1+d R \wedge * d R .
$$

Thus, (101) is equivalent to the Lagrangian density $R \Delta R * 1$ and consequently they lead to the same field equations. On the other hand, it is easy to show that the field equations that follow from $f(R \Delta R) * 1$ are not equivalent to those that follow from $f(*(d R \wedge * d R)) * 1$.

\section{CONCLUDING COMMENTS}

An apparent advantage of the use of the first order formalism is that it allows one to treat various modified gravitational Lagrangians ranging Ricci-squared Lagrangian in Palatini formalism and $f(R)$ models with nonmetricity and torsion to modified curvature-squared Lagrangians in a unified framework. From a technical point of view, it is also possible to relate the first order formalism to the C-theories introduced recently in [40]. In addition, the use of exterior algebra of tensorvalued forms renders the tensorial manipulations easier compared to the methods involving corresponding tensor components.

A general modified gravitational Lagrangian of the form $f(R, P, Q, K, S) * 1$ can be reduced to simpler Lagrangians involving $R, P, Q, K, S, \ldots$ with a number of nonminimally coupled scalar fields in the sense that $f(R) * 1$ is related to the Einstein-Hilbert Lagrangian $R * 1$ with nonminimally coupled scalar fields. The dynamical degree of freedom for the scalar fields, as well as the number of resulting independent scalar fields depend on the explicit form of the function $f$ as discussed with the explicit examples.

The multi-scalar-tensor equivalence discussed above for the modified gravitational actions can be regarded as an extension of the equivalence between the Brans-Dicketype ST theory and $f(R)$ theory to more complicated gravitational Lagrangians. In this regard, the procedure generates Brans-Dicke type scalar fields each of which are nonminimally coupled to curvature components. On the other hand $f(R)$ models are simple enough to accommodate a general connection with algebraic (i.e. nonpropagating) torsion and nonmetricity. These non-Riemannian models can also be cast into a form expressed in terms of pseudo-Riemannian quantities in addition to new gravitational interactions induced by non-Riemannian parts [10]. These features of $f(R)$ models, in general, do not carry over to modified gravitational Lagrangians based on more complicated curvature scalars.

The important issues to be addressed in constructing multi-ST equivalents are the number of resulting scalar fields and the determination of the dynamical degrees of freedom by the resulting scalar fields. The former problem has been addressed in 23] by using constraint analysis and the theory of primary constraints related to the Legendre transform of constrained systems. The latter issue can be addressed by discussing the ST equivalents at the level of field equations as we have discussed above. In the case where the scalar field is nondynamical ST equivalence becomes a superficial field redefinition. Moreover, save for some possible special cases, the scalar field satisfies a field equation of its own with certain curvature interaction terms depending on the particular curvature scalars under consideration.

With the experience gained through the ST equivalents for the gravitational models explicitly discussed above, the scope of the ST equivalence can be expanded 
to cover topological terms in gravitational models. In fact, the application of the idea of ST equivalence to topological terms provides a mechanism to incorporate such terms into gravitational Lagrangians [31]. Consider, for example, the Chern-Simons modified gravity where the Einstein-Hilbert Lagrangian is supplemented with a term $\Omega^{\alpha}{ }_{\beta} \wedge \Omega^{\beta}{ }_{\alpha}$ multiplied by a so-called cosmic scalar field. It is well known that, without a multiplicative scalar field, such a term does not contribute to the field equations, since it is the exterior derivative of the term $d \omega^{\alpha}{ }_{\beta} \wedge \omega^{\beta}{ }_{\alpha}+\frac{2}{3} \omega^{\alpha}{ }_{\beta} \wedge \omega^{\beta}{ }_{\mu} \wedge \omega^{\mu}{ }_{\alpha}$. As for all the examples studied above, one starts with the scalar $T$ which is defined by $T * 1 \equiv \Omega^{\alpha}{ }_{\beta} \wedge \Omega^{\beta}{ }_{\alpha}$, the Lagrangian density of the form $f(T) * 1$ with arbitrary function $f$ contributes to the field equations without the need to introduce a scalar field. In the same manner, in four dimensions, it is possible to incorporate quadratic Gauss-Bonnet term $G * 1 \equiv \Omega_{\alpha \beta} \wedge \Omega_{\mu \nu} \wedge * \theta^{\alpha \beta \mu \nu}$ into a gravitational Lagrangian by considering the term of the form $f(G) * 1$ see, for example, [31]. However, in neither of the cases the scalar field is dynamical.

\section{ACKNOWLEDGMENT}

It is a pleasure to dedicate the paper to Professor Metin Arlk on the occasion of his 65 th birthday.

\section{APPENDIX}

In order to facilitate comparison with the existing literature, often involving expressions and computations relative to a coordinate coframe, an illustration of how field equations relative to a coordinate coframe can be derived from the corresponding equations relative to an orthonormal coframe will be presented in some detail.

Any of the field equations $* E_{\alpha}=0$ derived above in terms of the vector-valued form $E_{\alpha}=E_{\alpha \beta} \theta^{\beta}$ is related to the coordinate expression $E_{a b}=0$ by $E_{\alpha \beta}$ with $E_{\alpha \beta}=$ $e_{\alpha}^{a} e_{\beta}^{b} E_{a b}$. (Here the Latin indices refer the to components of tensors relative to a coordinate coframe). Likewise, the basis coframe 1-forms are related by $\theta^{\alpha}=e_{a}^{\alpha} d x^{a}$ whereas the basis frame fields are related by $e_{\alpha}=e_{\alpha}^{a} \partial_{a}$ with $e_{a}^{\alpha} e_{\beta}^{a}=\delta_{\beta}^{\alpha}$ and $e_{a}^{\alpha} e_{\alpha}^{b}=\delta_{b}^{a}$.

After these preliminary definitions, take, for example the metric vacuum field equations $* E^{\alpha}=0$ for the $f(R)$ model, (21) derived above relative to an orthonormal coframe. These can conveniently be rewritten in the form

$$
-*\left(f^{\prime} R^{\alpha}-\frac{1}{2} f \theta^{\alpha}\right)+D *\left(d f^{\prime} \wedge \theta^{\alpha}\right)=0 .
$$

The derivation of the corresponding coordinate expressions then involves converting covariant exterior derivatives $D$ in the second term into covariant derivatives $\nabla_{e_{a}} \equiv \nabla_{a}$. This requires careful use of the properties of the covariant exterior derivative. In that, note that although $\nabla_{a}$ commutes with the Hodge dual operator *, $D$ does not commute with it. In particular, this term can be put into a more common form by using the operator identity,

$$
D i_{a}+i_{a} D=\nabla_{a},
$$

acting on an arbitrary $p$-form. For a derivation of this crucial identity as well as other properties of the covariant exterior derivative, the reader is referred to e.g. [27].

In order to make use of the identity (113), it is convenient to write the second term in the form $D *\left(d f^{\prime} \wedge \theta^{\alpha}\right)=$ $D i^{\alpha} * d f^{\prime}$. Here, another identity $i_{e^{\alpha}} * \sigma=*\left(\sigma \wedge \theta^{\alpha}\right)$ is used. This identity involving Hodge dual and the contraction operator holds for an arbitrary $p$-form $\sigma$ and the basis frame fields with a superscript are defined by $e^{\alpha}=\eta^{\alpha \beta} e_{\beta}$. Consequently, one ends up with

$$
D *\left(d f^{\prime} \wedge \theta_{a}\right)=\nabla_{a} * d f^{\prime}-i_{a}\left(d * d f^{\prime}\right),
$$

where the property that the covariant exterior derivative acting on an arbitrary form reduces to the exterior derivative has been used. Finally, by noting that covariant derivative of a Riemannian connection commutes with the Hodge dual operator $*$, which explicitly be stated as

$$
\nabla_{\alpha} *=* \nabla_{\alpha},
$$

and that $\Delta f^{\prime} \equiv * d * d f^{\prime}$, the second term simplifies to

$$
D *\left(d f^{\prime} \wedge \theta_{\alpha}\right)=*\left[\nabla_{\alpha} d f^{\prime}-\left(\Delta f^{\prime}\right) \theta_{\alpha}\right] .
$$

On the other hand, it follows from the definition of covariant derivative that $\nabla_{e_{\alpha}}=e_{\alpha}^{a} \nabla_{\partial_{a}}$. Consequently, it is possible to read off the coordinate expressions from the following expression

$$
D *\left(d f^{\prime} \wedge \theta_{\alpha}\right)=e_{\alpha}^{a} *\left[\nabla_{a} d f^{\prime}-\left(\Delta f^{\prime}\right) g_{a b} d x^{b}\right],
$$

since the expression in front of the Hodge dual now involves tensorial quantities relative to a coordinate coframe.

Eventually, this result allows one to extract $E_{a b}=0$ from the expression $E_{a b} * d x^{b}=0$ which simply follows from $* E_{a}=e_{a}^{\alpha} * E_{\alpha}$. Explicitly, by dropping the irrelevant Hodge dual, the resulting equations read

$$
E_{a b}=f^{\prime} R_{a b}-\frac{1}{2} g_{a b} f-\nabla_{a} \nabla_{b} f^{\prime}+g_{a b} \nabla^{c} \nabla_{c} f^{\prime}=0,
$$

relative to a coordinate frame. Evidently, these equations can directly be derived from the invariant Lagrangian density

$$
f(R) * 1=f(R) \sqrt{|g|} d x^{0} \wedge d x^{1} \wedge \cdots \wedge d x^{n},
$$

where $* 1=\theta^{012 \cdots n}=|e| d x^{01 \cdots n}=\sqrt{|g|} d x^{01 \cdots n}$ is the oriented volume element relative to a coordinate basis. These results also allow one to write the coordinate expression for the field equations of the ST equivalent of (118) immediately in the form

$$
\phi G_{a b}-\nabla_{a} \nabla_{b} \phi+g_{a b} \nabla^{c} \nabla_{c} \phi-\frac{1}{2} g_{a b} V(\phi)=0 .
$$


These metric equations follow from the following invariant Lagrangian density

$$
L_{S T}=\left(\phi g^{a b} R_{a b}-V(\phi)\right) \sqrt{|g|} d x^{0} \wedge d x^{1} \cdots \wedge d x^{n}
$$

where $R_{a b}$ are the components of the Ricci tensor relative to a coordinate coframe.

The field equations for the $f(R)$ model with nonmetricity and torsion also have similar terms and the corresponding coordinate expressions can be written in a similar way. Most of the identities used in the above calculations in deriving coordinate coframe expressions for a given modified gravitational Lagrangian are also useful in deriving coordinate expressions involving the Lagrange multiplier terms as well.

Likewise, in Section IV, the field equations for the sixth order gravity based on the Lagrangian density

$$
d R \wedge * d R=g^{a b} \partial_{a} R \partial_{b} R \sqrt{|g|} d x^{1} \wedge \cdots \wedge d x^{n},
$$

is considered where the coordinate expression on the right-hand side follows from the exterior derivative $d R=$ $\partial_{a} R d x^{a}$ and consequently the contraction of the basis coframe 1-forms as

$$
d x^{a} \wedge * d x^{b}=g^{a b} \sqrt{|g|} d x^{1} \wedge \cdots \wedge d x^{n} .
$$

It is possible to derive the coordinate expression for the sixth order model by making use of the expression $E_{a b}$ for the $f(R)$ model since the corresponding field equations are cast in a form that is formally similar to those of the $f(R)$ model. It is sufficient to derive the coordinate expression for the vector-valued form $* T_{s}^{\alpha}$ defined in (103), which can be rewritten in the form as

$$
\begin{aligned}
* T_{s}^{\alpha} & =\left(i^{\alpha} d R\right) * d R+i^{\alpha}(d R \wedge * d R) \\
& =e_{a}^{\alpha}\left[2\left(i^{a} d R\right) * d R-i^{a}(d R \wedge * d R)\right],
\end{aligned}
$$

and therefore, by recalling that $i_{a} d R=\partial_{a} R$ relative to a coordinate coframe, one can read off the coordinate expression for the energy momentum 1-form as

$$
T_{a}^{s}=2 \partial_{a} R d R-g_{a b} g^{c d} \partial_{c} R \partial_{d} R d x^{b}
$$

and in turn, using this result, the coordinate expression for $T_{a b}^{s}$ can be obtained as

$$
T_{a b}^{s}=2 \partial_{a} R \partial_{b} R-g_{a b} g^{c d} \partial_{c} R \partial_{d} R .
$$

This result then allows one to write the explicit form of $E_{a b}=0$ for the sixth order model as

$$
\begin{aligned}
& F R_{a b}-\frac{1}{2} g_{a b} F-\nabla_{a} \nabla_{b} F+g_{a b} \nabla^{c} \nabla_{c} F \\
& -\partial_{a} R \partial_{b} R+\frac{1}{2} g_{a b} g^{c d} \partial_{c} R \partial_{d} R=0
\end{aligned}
$$

with $F=\Delta R=g^{a b} \nabla_{a} \nabla_{b} R$ replacing the function $f^{\prime}$ for the $f(R)$ model as a consequence of the identity $d \Delta=$ $\Delta d$. For the coordinate expression for the ST equivalents related to the sixth order model, the reader is referred to the original work [55].

The modified-QC gravity field equations are based on the quadratic curvature invariants of the form

$$
L=f\left(R^{2}, R_{a b} R^{a b}, R_{a b c d} R^{a b c d}\right) \sqrt{|g|} d x^{0} \wedge \cdots \wedge d x^{n},
$$

and under certain assumptions about the rank of relevant Legendre transformation, the ST equivalents for this Lagrangian have the general form

$$
\begin{aligned}
L_{S T}= & {\left[\phi_{1} R^{2}+\phi_{2} R_{a b} R^{a b}+\phi_{3} R_{a b c d} R^{a b c d}\right.} \\
& \left.-V\left(\phi_{1}, \phi_{2}, \phi_{3}\right)\right] \sqrt{|g|} d x^{0} \wedge \cdots \wedge d x^{n},
\end{aligned}
$$

where the independent scalar fields $\phi_{1}, \phi_{2}$ and $\phi_{3}$ are defined in (67) whereas the potential term $V\left(\phi_{1}, \phi_{2}, \phi_{3}\right)$ is the Legendre transform of $f\left(R^{2}, R_{a b} R^{a b}, R_{a b c d} R^{a b c d}\right)$ given in (69). In particular, for the simple modified QC Lagrangians having the particular form

$$
L=\left(a R^{2}+b R_{a b} R^{a b}+c R_{a b c d} R^{a b c d}\right)^{m} \sqrt{|g|} d x^{0} \wedge \cdots \wedge d x^{n},
$$

there is an ST equivalent with potential for the scalar of the form of a power law

$$
\begin{aligned}
L_{S T}= & \phi \phi\left(a R^{2}+b R_{a b} R^{a b}+c R_{a b c d} R^{a b c d}\right) \\
& \left.-\left(\frac{1-m}{m}\right) \phi^{m / m-1}\right] \sqrt{|g|} d x^{0} \wedge \cdots \wedge d x^{n} .
\end{aligned}
$$

In general QC field equations involve the terms of the form $D * D L^{a}$, where 1 -form $L^{a}$ is typically linear combination of Ricci 1-form and the 1-form $R d x^{a}$. Take, for example, the Weyl-squared action in four dimensions. It follows from the invariant Lagrangian density

$$
\begin{aligned}
C^{\alpha \beta} \wedge * C_{\alpha \beta} & \equiv C^{a b} \wedge * C_{a b} \\
& =\frac{1}{2} C^{a b}{ }_{c d} C_{a b}{ }^{c d} \sqrt{|g|} d x^{0} \wedge \cdots \wedge d x^{3} .
\end{aligned}
$$

The corresponding field equations can be expressed as the vanishing of the Bach tensor $E_{a b}^{W} \equiv-B_{a b}=0$. An explicit expression for the coordinate components of Bach tensor turns out to be

$$
B_{a b}=\nabla^{c} C_{a c b}+R^{c d} C_{a c b d}
$$

where $C_{a c b}$ are the components of the Cotton 2-form [42] defined by $C_{a}=\frac{1}{2} C_{a b c} d x^{b} \wedge d x^{c}$ whereas $C_{b c d}^{a}$ are components of Weyl tensor $C^{a}{ }_{b}=\frac{1}{2} C^{a}{ }_{b c d} d x^{b} \wedge d x^{c}$. Moreover, for the divergence of the Cotton 2-form, using the identity (113) one finds

$$
\begin{aligned}
D * C^{a} & =\frac{1}{2} D C_{b c}^{a} \wedge *\left(d x^{b} \wedge d x^{c}\right) \\
& =\frac{1}{2} \nabla_{d} C_{b c}^{a} d x^{d} \wedge *\left(d x^{b} \wedge d x^{c}\right) .
\end{aligned}
$$


On the other hand, the contraction on the right-hand side leads to the following Hodge dual of basis 1 -forms

$$
d x^{d} \wedge *\left(d x^{b} \wedge d x^{c}\right)=-g^{b d} * d x^{c}+g^{c d} * d x^{b} .
$$

Using this result, it is easy to find

$$
D * C_{a}=-\nabla^{b} C_{a b c} * d x^{c}
$$

The Cotton 2-form itself can be expressed in terms of the covariant derivatives of curvature components. It is derived from Schouten tensor $C^{a}=D L^{a}$ where the Schouten tensor $L_{a b}$ can be defined in terms of $L_{a} \equiv$
$L_{a b} d x^{b}$ as $L_{a b}=R_{a b}-\frac{1}{6} g_{a b} R$ (the definitions in the literature vary up to an overall constant). By taking the antisymmetrization induced by the covariant exterior derivative into the account, the coordinate components of the Cotton 2-form for example take the form

$$
C_{a b c}=\nabla_{a} R_{b c}-\nabla_{b} R_{a c}-\frac{1}{6}\left(\nabla_{a} R g_{b c}-\nabla_{b} R g_{a c}\right) \text {. }
$$

Evidently, these formulas also help to relate orthonormal components of QC gravity to the corresponding ones relative to a coordinate basis. For a more detailed account for coordinate components of a given expression relative to an orthonormal coframe, the reader is referred to, for example, 42.
[1] T. Clifton, P. G. Ferreira, A. Padilla, and C. Skordis, Phys. Rep. 513, 1 (2012); S. Nojiri and S. D. Odintsov, Phys. Rep. 505, 59 (2010).

[2] T. P. Sotiriou and V. Faraoni, Rev. Mod. Phys. 82, 451 (2010).

[3] B. Zwiebach, Phys. Lett. B 156, 315 (1985); A. Tseytlin, Phys. Lett. B 176, 92 (1986).

[4] K. S. Stelle, Phys. Rev. D 16, 953 (1977); R. Utiyama and B. S. DeWitt, J. Math. Phys. (N.Y.) 3, 608 (1962); K. S. Stelle, Gen. Relativ. Gravit. 9, 353 (1978).

[5] A. Starobinsky, Phys. Lett. B 91, 99 (1980).

[6] H -J Schmidt, Int. J. Geom. Meth. Mod. Phys. 4, 209 (2007).

[7] B. Whitt, Phys. Lett. B 145, 176 (1984).

[8] P. Teyssandier and Ph. Tourrenc, J. Math. Phys. (N. Y.) 24, 2793 (1983).

[9] C. Brans and R. H. Dicke, Phys. Rev. 124, 925 (1961).

[10] T. Dereli and R. W. Tucker, Phys. Lett. B 110, 206 (1982).

[11] T. P. Sotiriou, Class. Quantum Grav. 26, 152001 (2009); T. P Sotiriou, Class. Quantum Grav. 23, 5117 (2006).

[12] J. Khoury and A. Weltman, Phys. Rev. Lett. 93, 171104 (2004); J. Khoury and A. Weltman, Phys. Rev. D 69, 044026 (2004).

[13] S. Capozziello and D. Saez-Gomez, Ann. Phys. (N. Y.) 524, 279 (2012).

[14] V. Faraoni, Phys. Rev. D 81, 044002 (2010).

[15] S. Capozziello, C. Corda, and M. F. De Laurentis, Phys. Lett. B 669, 255 (2008).

[16] G. Magnano, M. Ferraris, and M. Francaviglia, Gen. Relativ. Gravit. 19, 465 (1987).

[17] G. Magnano and L. M. Sokołowski, Phys. Rev. D 50, 5039 (1994).

[18] G. Magnano, M. Ferraris, and M. Francaviglia, Class. Quantum Grav. 7, 557 (1990).

[19] È. È. Flanagan, Class. Quantum Grav. 21, 417 (2004).

[20] I. Saltas and M. Hindmarsh, Class. Quantum Grav. 28, 035002 (2011).

[21] S. Nojiri and S. D. Odintsov, Phys. Lett. B 631, 1 (2005).

[22] T. Chiba, JCAP03 (2005) 008.

[23] D. C. Rodrigues, F. O. Salles, I. L. Shapiro, and A. A. Starobinsky, Phys. Rev. D 83, 084028 (2011).

[24] R. Kerner, Gen. Relativ. Gravit. 14, 453 (1982).

[25] S. M. Carroll, A. De Felice, V. Duvvuri, D. A. Easson,
M. Trodden, and M. S. Turner, Phys. Rev. D 71, 063513 (2005)

[26] N. Straumann, General Relativity with Applications to Astrophysics, 2nd. Ed. (Springer, Dordrecht, 2013).

[27] I. M. Benn and R. W. Tucker, An Introduction to Spinors and Geometry With Applications in Physics (Adam Hilger, Bristol, UK, 1988)

[28] F. W. Hehl, J. D. McCrea, E. W. Mielke, and Y. Ne'eman, Phys. Rep. 258, 1 (1995).

[29] W. Kopczyǹsky, Ann. Phys. (N.Y.), 203, 308 (1990).

[30] T. P. Sotiriou, Class. Quantum Grav. 26, 152001 (2009).

[31] A. Baykal and Ö. Delice, Class. Quantum Grav. 28, 015014 (2011).

[32] A. Baykal, Gen. Relativ. Gravit. 44, 1993 (2012).

[33] T. Dereli and R. W. Tucker, Class. Quantum Grav. 4, 791 (1987).

[34] Yuri N. Obukhov and G. F. Rubilar, Phys. Rev. D 74, 064002 (2006).

[35] S. Kichenassamy, Ann. Phys. (N.Y.) 168, 404 (1986).

[36] A. Baykal and Ö. Delice, Gen. Relativ. Gravit. 41, 267 (2009).

[37] J. O'Hanlon, Phys. Rev. Lett. 29, 137 (1972).

[38] T. Chiba, Phys. Lett. B 575, 1 (2003).

[39] T. Dereli and R. W. Tucker, Class. Quantum Grav. 12, L31 (1995).

[40] L. Amendola, K. Enqvist, and T. Koivisto, Phys. Rev. D 83, 044016 (2011).

[41] Tomi S. Koivisto, David F. Mota, and Marit Sandstad, arXiv:1305.4754

[42] A. Garcia, F. W. Hehl, C. Heinicke, and A. Macias, Class. Quantum Grav. 21, 1099 (2004).

[43] C. Lanczos, Ann. Math. (N.Y.) 39, 842 (1938); D. Lovelock, Proc. Cambridge Philos. Soc. 68, 345 (1970).

[44] W. Thirring, Classical Mathematical Physics: Dynamical Systems and Field Theories, 3rd Ed. (Springer-Verlag, New York, 1997).

[45] A. Jakubiec and J. Kijowski, Phys. Rev. D 37, 1406 (1988).

[46] A. Borowiec, M. Ferraris, M. Francaviglia, and I. Volovich, Class. Quantum Grav. 15, 43 (1998).

[47] A. Borowiec, M. Ferraris, M. Francaviglia, and I. Volovich, Class. Quantum Grav. 11, 1505 (1994).

[48] M. P. Dabrowski, J. Garecki, and D. B. Blaschke, Ann. Phys. (Paris) 18, 13 (2009). 
[49] S. M. Carroll, V. Duvvuri, M. Trodden, and M. S. Turner, Phys. Rev. D 70, 043528 (2004).

[50] R. Bach, Math. Z. 9, 110 (1921).

[51] T. Dereli and R. W. Tucker, J. Phys. A 15, L7 (1982).

[52] V. P. Frolov and I. L. Shapiro, Phys. Rev. D 80, 044034 (2009).
[53] T. Biswas, A. Mazumdar, and W. Siegel, JCAP 03 (2006) 009.

[54] J. Hwang, Class. Quantum Grav. 8, L133 (1991).

[55] S. Gottlöber, H.-J. Schmidt, and A. A. Starobinsky, Class. Quantum Grav. 7, 893 (1990). 